\title{
The mitochondrial citrate carrier in Yarrowia lipolytica: Its identification, characterization and functional significance for the production of citric acid
}

\author{
Evgeniya Y. Yuzbasheva ${ }^{\mathrm{a}, * *, 1}$, Gennaro Agrimi $^{\mathrm{b}, 1}$, Tigran V. Yuzbashev ${ }^{\mathrm{c}}$, Pasquale Scarcia ${ }^{\mathrm{b}}$, \\ Elizaveta B. Vinogradova $^{a}$, Luigi Palmieri ${ }^{\mathrm{b}, \mathrm{d}}$, Artem V. Shutov ${ }^{\mathrm{a}}$, Iuliia M. Kosikhina ${ }^{\mathrm{a}}$, \\ Ferdinando Palmieri ${ }^{\mathrm{b}, \mathrm{d}, *}$, Sergey P. Sineoky ${ }^{\mathrm{a}}$ \\ ${ }^{a}$ Bioresource Center Russian National Collection of Industrial Microorganisms (BRC VKPM), State Research Institute of Genetics and Selection of Industrial \\ Microorganisms National Research Centre "Kurchatov Institute" (NRC "Kurchatov Institute" - GosNIIgenetika), 1-st Dorozhny pr., 1, Moscow, 117545, Russian Federation \\ ${ }^{\mathrm{b}}$ Department of Biosciences, Biotechnologies and Biopharmaceutics, University of Bari “Aldo Moro", Campus Universitario, Via Orabona 4, 70125, Bari, Italy \\ ${ }^{\mathrm{c}}$ Department of Bioengineering, Imperial College London, London, SW7 2AZ, UK \\ ${ }^{\mathrm{d}}$ CNR Institute of Biomembranes, Bioenergetics and Molecular Biotechnologies (IBIOM), Campus Universitario, Via Orabona 4, 70125, Bari, Italy
}

\section{A R T I C L E I N F O}

\section{Keywords:}

Yarrowia lipolytica

Mitochondrial citrate carrier

YlYhm2p

Citric acid production

\begin{abstract}
A B S T R A C T
Mitochondrial citrate carrier plays a central role in exporting acetyl-CoA in the form of citrate from mitochondria to cytosol thereby connecting carbohydrate catabolism and lipogenesis. In this study, Yarrowia lipolytica mitochondrial citrate carrier was functionally defined and characterized. Firstly, deletion of $Y$. lipolytica YlCTP1 and YlYHM2 genes coding putative tricarboxylate mitochondrial carriers were performed. $\Delta Y l c t p 1$ strain did not differ significantly from wild type strain in terms of growth rate, organic acids and lipid production. In contrast, $\triangle Y$ Ylyhm2 strain did not grow in liquid citrate-containing minimal medium. Moreover, in glucosecontaining lipogenic medium YlYHM2 null mutant strain did not produce citric acid; the production of isocitric acid and lipids were decreased. Reintroduction of YlYHM2 gene as well as heterologous expression of Aspergillus niger gene AnYHM2 into $\triangle Y$ Ylyhm2 strain restored the growth in minimal citrate medium and even enhanced citric acid production by $45 \%$ in both variants compared with wild type strain during test tube cultivation. Mitochondrial extracts isolated from YlYHM2 null mutant and wild type strain were incorporated into liposomes; citrate/citrate and $\alpha$-ketoglutarate/ $\alpha$-ketoglutarate homoexchange activities were reduced by $87 \%$ and $40 \%$ in $\Delta$ Ylyhm2 strain, respectively, compared with the wild type, whereas citrate $_{\text {in }} / \alpha$-ketoglutarate ${ }_{\text {out }}$ and $\alpha$-ketoglutarate $_{\text {in }} /$ citrate $_{\text {out }}$ heteroexchanges were decreased by $87 \%$ and $95 \%$, respectively. YlYhm2p was expressed in Escherichia coli, purified and reconstituted into liposomes. Besides high efficiency to citrate and $\alpha$-ketoglutarate transport, YlYhm2p also transported oxaloacetate, succinate, fumarate, and to a much lesser extent, aconitate, malate, isocitrate, oxoadipate, and glutamate. The activity of reconstituted YlYhm2p was inhibited strongly by SH-blocking reagents, pyridoxal-5'-phosphate, and partly by N-ethylmaleimide. Co-expression of YlYHM2 and adenosine monophosphate deaminase YlAMPD genes resulted in the production of $49.7 \mathrm{~g} / \mathrm{L}$ of citric acid during test tube cultivation, whereas wild type strain accumulated $30.1 \mathrm{~g} / \mathrm{L}$ of citric acid. Large-scale cultivation in bioreactor of the engineered strain resulted in $97.1 \mathrm{~g} / \mathrm{L}$ of citric acid production with a process selectivity of $94.2 \%$ and an overall citric acid yield of $0.5 \mathrm{~g} / \mathrm{g}$. The maximal specific rate of citric acid synthesis was $0.93 \mathrm{~g} / \mathrm{L} / \mathrm{h}$. Therefore, the physiological role of YlYhm2p in glucose-containing medium is to catalyze both import of citrate into mitochondria for catabolic reactions and export of citrate as a source of acetyl-CoA from mitochondria. Possible shuttles for citrate exporting are discussed. Moreover, for the first time evidence has been given for the improvement of TCA cycle intermediate production by manipulation of a gene coding a mitochondrial carrier.
\end{abstract}

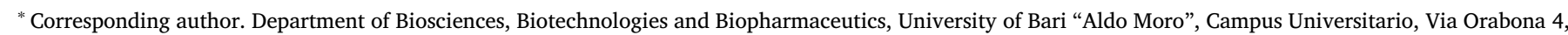
70125, Bari, Italy

${ }^{* *}$ Corresponding author. Bioresource Center Russian National Collection of Industrial Microorganisms (BRC VKPM), State Research Institute of Genetics and

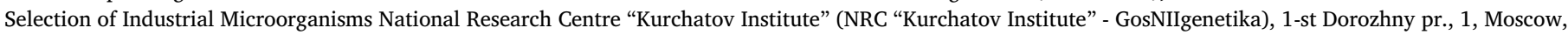
117545, Russian Federation.

E-mail addresses: enja_t@mail.ru (E.Y. Yuzbasheva), ferdpalmieri@gmail.com (F. Palmieri).

1 - These authors contributed equally to this work. 

Abbreviations:
TCA tricarboxylate cycle
AMPD adenosine monophosphate deaminase
MC mitochondrial carrier
CIC mitochondrial citrate carrier
DTC dicarboxylate-tricarboxylate mitochondrial carrier

\section{Introduction}

Yarrowia lipolytica is "non-conventional" oleaginous yeast that serves as a relevant platform for enzyme technology, organic acid production and lipid accumulation (Liu et al., 2015; Madzak, 2015; Nicaud, 2012). Depending on the cultivation conditions natural isolates of $Y$. lipolytica are able to secrete high amounts of tricarboxylic acid cycle (TCA) intermediates, such as pyruvic, citric, isocitric or $\alpha$-ketoglutaric acids (Barth and Gaillardin, 1997). Classical mutation breeding as well as rational design of strains by gene modifications and metabolic engineering have been successfully applied to enhance the production of organic acids (Blazeck et al., 2015; Guo et al., 2016; Morgunov et al., 2013; Yuzbashev et al., 2010). Using these strategies, a substantial improvement in lipid accumulation by $Y$. lipolytica has also been achieved (Ledesma-Amaro et al., 2016; Qiao et al., 2017; Xu et al., 2016). During nitrogen starvation and excess of glucose as a carbon source $Y$. lipolytica accumulates neutral lipids and produces a mixture of citric and isocitric acids. Under these conditions ammonium is produced from AMP through the activity of adenosine monophosphate deaminase (AMPD) (Beopoulos et al., 2011), and AMP is an allosteric activator of mitochondrial isocitrate dehydrogenase. Hence, a decrease in the AMP pool regulates isocitrate dehydrogenase negatively and results in accumulation of citrate and isocitrate in mitochondria.

The inner membrane of mitochondria is impermeable to organic acids. However, this membrane is equipped with a family of mitochondrial carrier (MC) proteins, named mitochondrial carrier family, that are responsible for the translocation of specific metabolites, such as inorganic anions, di- and tri-carboxylates, keto acids, amino acids, nucleotides and coenzymes (Krämer and Palmieri, 1992; Palmieri, 2013, 2014). The protein sequences of the MC family members have a characteristic three times tandemly repeated 100 residue domain, which contains two hydrophobic segments and a signature sequence motif PX [D/E]XX [K/R]X [K/R] (20-30 residues) [D/E]GXXXX [W/Y/ F][K/R]G (PROSITE PS50920, PFAM PF00153 and IPR00193) (Palmieri, 1994). In atomic resolution 3D-structures of the only MC family member determined until now (the carboxyatractyloside-inhibited ADP/ATP carrier) (Pebay-Peyroula et al., 2003; Ruprecht et al., 2014) the six hydrophobic segments form a bundle of transmembrane $\alpha$-helices with a central substrate translocation pore, and the three PX [D/E]XX [K/R] motifs form a gate towards the matrix side. In most cases, the MC distinct sequence features and particularly their signature motif have been used to identify family members in genomic sequences. Among the numerous MCs, the citrate carrier is generally believed to be essential for fatty acid and sterol biosynthesis, given that it exports citrate from the mitochondria to the cytosol, where it is cleaved by ATPcitate lyase to oxaloacetate and acetyl CoA, which is used for fatty acid and sterol biosynthesis (Palmieri, 2004).

The mitochondrial citrate carrier (CIC) has been identified and characterized first in rat liver (Bisaccia et al., 1989; Kaplan et al., 1990; Palmieri et al., 1972). In the reconstituted system, CIC catalyses an electroneutral, obligatory exchange of the dibasic form of a tricarboxylic acid (e.g. citrate, isocitrate, and cis-aconitate) for another tricarboxylate $/ \mathrm{H}^{+}$, a dicarboxylate (malate, succinate and malonate) or phosphoenolpyruvate (Bisaccia et al., 1990, 1993). In plants, a related carrier to CIC is the dicarboxylate-tricarboxylate carrier (DTC) that transports both dicarboxylates (malate, oxaloacetate, oxoglutarate and maleate) and tricarboxylates (citrate, isocitrate, cis-aconitate and transaconitate) (Picault et al., 2002). Among yeasts the MC for citrate Ctp1p has been partially characterized in terms of substrate specificity only in $S$. cerevisiae traditionally served as a model yeast; it translocates citrate efficiently and, to a much lesser extent, only isocitrate and phosphoenolpyruvate (Kaplan et al., 1995). Notably, S. cerevisiae and other fermentative yeasts lack ATP-citrate lyase activity, thereby they cannot use citrate as a source of acetyl-CoA (Boulton and Ratledge, 1981). Indeed, fermentative yeasts generate cytosolic acetaldehyde that is converted to cytosolic acetyl-CoA by pyruvate decarboxylase, aldehyde dehydrogenase, and acetyl-CoA synthetase (Pronk et al., 1994). In $S$. cerevisiae a second MC for citrate, Yhm2p, has been functionally defined; it transports citrate and $\alpha$-ketoglutarate with high efficiency and oxaloacetate, succinate, fumarate and $\alpha$-ketoadipate to a lesser extent, but virtually not malate and isocitrate (Castegna et al., 2010). This carrier has been shown to increase the NADPH reducing power in the cytosol and to act as a key component of the citrate/ $\alpha$-ketoglutarate NADPH redox shuttle between mitochondria and cytosol (Castegna et al., 2010). Yhm2p has also been demonstrated to transport $\alpha$-ketoglutarate from mitochondria to the cytosol for the synthesis of glutamate and ammonium fixation in cytosol as well as to export $\alpha$-ketoadipate for the synthesis of lysine (Scarcia et al., 2017).

Despite significant advancement in understanding the physiology, genetics, and metabolism of $Y$. lipolytica as well as in engineering new recombinant strains via synthetic biology, little is known about MC proteins in this yeast. Thus, in Y. lipolytica only the three genes for mitochondrial ADP/ATP carriers have been found to be regulated differentially in the absence of oxygen (Mentel et al., 2005), and an uncoupling-like activity of a putative MC for oxaloacetate has been described (Luevano-Martinez et al., 2010). Identification and characterization of MC proteins able to transport carboxylic acids in $Y$. lipolytica is an important objective that can pave the way to improve titers of organic acid production and lipids through strain genetic engineering.

In this study, citrate mitochondrial carrier in oleaginous yeast has been identified and characterized for the first time. For this purpose, firstly deletion of two genes coding putative tricarboxylate mitochondrial carriers in $Y$. lipolytica was performed. Unexpectedly, deletion of YALIOF26323g gene (designated as YlCTP1) coding the closest homolog of $S$. cerevisiae Ctp1p did not have any influence on growth as well as on citric and isocitric acids production and de novo lipid accumulation in $Y$. lipolytica during cultivation on glucose-containing lipogenic medium. In contrast, YALIOB10736p protein (designated as YlYhm2p) which is the ortholog of $S$. cerevisiae Yhm2p played an essential role in the citrate transport across inner membrane in Y. lipolytica. YlYHM2 null mutant strain did not grow in minimal liquid medium with citrate as a single carbon source and it did not produce citric acid during cultivation on glucose-containing lipogenic medium. Reintroduction of homologous YlYHM2 gene as well as introduction of heterologous Aspergillus niger gene ANI_1_874084 (designated as AnYHM2) in $\triangle$ Ylyhm2 strain fully restored growth and citric acid production. Mitochondrial extracts isolated from constructed strains were incorporated into liposomes and were analyzed for citrate and $\alpha$-ketoglutarate exchange activity. YlYhm2p was expressed in Escherichia coli, purified, reconstituted into liposomes, and identified for its transport properties. Furthermore, substantial increase in citric acid production has been achieved by coexpression of YlYHM2 and YlAMPD.

\section{Materials and methods}

\subsection{Strains and growth media}

E. coli XL1-Blue was used for plasmid construction, propagation and amplification. E. coli M15 (pREP4) was used for YlCTP1 and YlYHM2 genes expression and proteins purification. Media and growth conditions for $E$. coli have been described elsewhere (Sambrook et al., 1989). 
Yeast minimal growth medium was YNBD $(0.67 \%$ yeast nitrogen base, Difco, USA) with $20 \mathrm{~g} / \mathrm{L}$ glucose. Yeast rich growth medium was YPD ( $10 \mathrm{~g} / \mathrm{L}$ yeast extract, $10 \mathrm{~g} / \mathrm{L}$ bacto-peptone and $20 \mathrm{~g} / \mathrm{L}$ glucose). Solid media were prepared by adding $20 \mathrm{~g} / \mathrm{L}$ agar. Uracil was supplemented at $50 \mu \mathrm{g} / \mathrm{mL}$ when necessary.

Growth curves of $Y$. lipolytica strains were monitored in $\mathrm{YNBD}_{0.8}$ and YNBCitr $_{0.8}$ (i. e. YNB supplemented with $8 \mathrm{~g} / \mathrm{L}$ glucose and citrate, respectively) using compact rocking incubator (Advantec MFS Inc., USA). Inoculates were grown in $5 \mathrm{~mL}$ YPD to early-stationary phase and transferred to the media to a final titre of $3 \times 10^{5}$ cells $/ \mathrm{mL}$.

The medium for cultivation (lipogenic medium) was $\mathrm{YNBN}_{0.2} \mathrm{D}_{9}$ consisting of YNB without ammonium sulphate (Difco, USA) but supplemented with $2 \mathrm{~g} / \mathrm{L}$ ammonium sulphate and $90 \mathrm{~g} / \mathrm{L}$ glucose. Cultivation was performed in $50 \mathrm{~mL}$ test tubes containing $10 \mathrm{~mL}$ of medium for 5 days at $30{ }^{\circ} \mathrm{C}$ on an orbital shaker operating at $250 \mathrm{rpm}$. Inoculates were grown in $5 \mathrm{~mL}$ YPD and transferred to cultivation media to a final titre of $1 \times 10^{5}$ cells $/ \mathrm{mL}$. The $\mathrm{pH}$ value was maintained at $4-5$ by adding sterile $1 \% \mathrm{CaCO}_{3}$. All strains were cultured in triplicate. As $\mathrm{CaCO}_{3}$ was used to maintain the $\mathrm{pH}$ value the crystals were solved by mixing culture broth with equal volume of $1.0 \mathrm{~N} \mathrm{HCl}$. To measure dry cell weight (dcw), $1 \mathrm{~mL}$ cell suspension was collected, washed and lyophilized for $24 \mathrm{~h}$ with FreeZone 6 Plus (Labconco, USA).

For the preparation of the mitochondria, the cells were grown on $\mathrm{YNBN}_{0.2} \mathrm{D}_{9}$ medium to mid-exponential phase. The mitochondria were isolated according to standard procedures (Palmieri et al., 1999b).

\subsection{ORF identification}

The genes coding for putative mitochondrial tricarboxylate carriers were identified by on-line TBLASTn search (http://blast.ncbi.nlm.nih. gov/). Query sequences of $S$. cerevisiae mitochondrial citrate carrier Ctp1p and mitochondrial citrate/ $\alpha$-ketoglutarate carrier Yhm2p were used for $Y$. lipolytica putative mitochondrial tricarboxylate carriers' identification. Only one open reading frame (ORF) was found for each query with high amino acid sequence identity: YALIOF26323g and YALIOB10736g encoding two proteins named YlCtp1p and YlYhm2p, respectively, in accordance with the previously used nomenclature in $S$. cerevisiae. The amino acid sequence of protein encoded by $A$. niger ANI_1_874084 locus tag was revealed by online search against $A$. niger genome with $S$. cerevisiae $Y h m 2 p$ as a query sequence. This protein was designated as AnYhm2p. The amino acid sequence of AnYhm2p shared 69.4 and $71.7 \%$ identical amino acids and 79.8 and $82.7 \%$ similarity with $S$. cerevisiae $\mathrm{Yhm} 2 \mathrm{p}$ and $Y$. lipolytica $\mathrm{YlYhm} 2 \mathrm{p}$, respectively. Sequences were aligned with ClustalW.

\subsection{Construction of plasmids}

Enzymes for molecular biology were obtained from Thermo Fisher Scientific (USA), and basic DNA manipulations were performed as described in Sambrook et al. (1989). Oligonucleotide primers are summarized in Supplementary Table S1. Y. lipolytica target nucleotide fragments were amplified using HifiCAPA-polymerase from strain W29 (ATCC, 20460). PCR products were verified by sequencing both strands.

Plasmids pUC-flYlCTP1-URA3 and pUC-flYlYHM2-URA3 were constructed to inactivate $Y$. lipolytica YlCTP1 and YlYHM2 genes, respectively. The nucleotide fragments of target genes and their upstream and downstream surrounding sequences were amplified using primer pairs YlCTP-SacI-F and YlCTP-Ecl136II-R, YlYHM2-EheI-F and YlYHM2-EheI$\mathrm{R}$, respectively, and cloned into pUC19 digested with Ecl136II. The central parts of YlCTP1 and YlYHM2 genes were then excised using $K p n 2 I$ and $B c l I / B s u 15 I$ endonucleases, respectively, and replaced with URA3 gene amplified with primers pair Lox66-URA3-F and Lox71URA3-R.

The plasmid pTEFin-uno-YlYHM2 for YlYHM2 gene over-expression was constructed as follows. Promoter TEFin was amplified using primers pair TEFin-NheI-F and TEFin-BpiI-R. PCR-product was treated with
NheI and BpiI, and introduced into the plasmid php4d-uno (Yuzbasheva et al., 2017) digested in the same way to generate the plasmid pTEFinuno. YlYHM2 gene was amplified using primers pair YlYHM2-Esp3I-F and YlYHM2-XmaJI-R, treated with Esp3I and XmaJI, and inserted into the BpiI and XmaJI sites in plasmid pTEFin-uno. The plasmid php4duno-YLAMPD for YLAMPD gene over-expression under hp4d promoter (Madzak et al., 2000) was constructed as follows. YlAMPD gene was obtained by assembling two PCR products amplified using the primer pairs YlAMPD-Aarl-hp4d-F and YlAMPD-less-R, and YLAMPD-less-F and YlAMPD-XmaJI-R. An internal AarI site in YLAMPD was eliminated in this manner. The amplicon was then digested with AarI and XmaJI, and introduced into the BpiI and XmaJI sites in plasmid php4d-uno. Plasmid pTEFin-uno-AnYHM2 was constructed for A. niger AnYHM2 gene overexpression. AnYHM2 gene was synthesized by Innova Plus (Russia). Rare codons in AnYHM2 were manually optimized according to Y. lipolytica codon usage (Supplementary Fig. S1). The synthesized fragment was treated with Esp3I and XmaJI and introduced into the plasmid pTEFin-uno digested with BpiI and XmaJI.

Plasmids pQE30-YlCTP1 and PQE30-YlYHM2 were constructed for YlCTP1 and YlYHM2 genes expression in E. coli and purification of proteins. The plasmids were constructed by the Gibson assembly method (Gibson et al., 2009). Rare codons in YlCTP1 and YlYHM2 were manually optimized according to $E$. coli codon usage (Supplementary Figs. S2 and S3). Given that YlCTP1 native gene contains an intron, two fragments of YlCTP1 were amplified using primers pairs YlCTP1-1-pQ-F and YlCTP1-1-R, YlCTP1-2-F and YlCTP1-2-pQ-R, respectively, and assembled with the vector part amplified from $\mathrm{pQE} 30$ using primers $\mathrm{pQE}$ $\mathrm{F}$ and $\mathrm{pQE}-\mathrm{R}$. YlYHM2 native gene contains two introns, thus the three fragments of YlYHM2 were amplified using primers pairs YlYHM2-1pQ-F and YlYHM2-1-R, YlYHM2-2-F and YlYHM2-2-R, and YlYHM2-3-F and YlYHM2-3-pQ-R, respectively, and assembled with the vector part amplified from pQE30.

\subsection{Construction of recombinant strains}

Yeast strains were transformed by electroporation (Yuzbasheva et al., 2015). All transformants were selected on YNBD medium by restoration of prototrophy for uracil. The disruption cassettes flYlCTP1URA3 and flYlYHM2-URA3 were released from the plasmids using SacI/ Ecl136II or EheI endonucleases, respectively, and transformed into W29 ( $\triangle$ ura3). PCRs using primers YlCTP1-chr-F and YlCTP1-chr-R, YlYHM2chr-F and YlYHM2-chr-R were applied to verify the correct chromosomal integration, and hence YlCTP1 and YlYHM2 genes disruption. Verified transformants were designated as W29 ( Ylctp1) and W29 $(\Delta$ Ylyhm2), respectively. The auxotrophic derivative W29 $(\Delta$ Ylyhm2 Ura-) was obtained using Cre-lox66/71 recombination system as described previously (Yuzbasheva et al., 2017).

Expression cassette $h p 4 d$-uno-YLAMPD was released from the plasmid php4d-uno-YlAMPD by digestion with Psp1406I/SspI endonucleases and transformed into the strain W29 ( $\triangle$ ura3) to obtain W29 (YlAMPD) strain. The auxotrophic derivative W29 (YLAMPD Ura-) was obtained using Cre-lox66/71 recombination system.

The expression cassette TEFin-uno-YlYHM2 was released from the plasmid pTEFin-uno-YlYHM2 by digestion with Cail and EheI en-

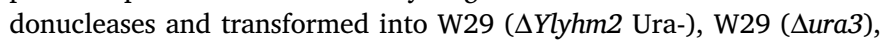
and W29 (YLAMPD Ura-) strains to obtain strains W29 ( $\triangle$ Ylyhm2 YlYHM2) and W29 (YlYHM2), and W29 (YlAMPD YlYHM2), respectively. The expression cassette TEFin-uno-AnYHM2 was released from the plasmid pTEFin-uno-AnYHM2 by digestion with SapI and EcoO109I, and electroporated into W29 ( $\triangle$ Ylyhm2 Ura-) and W29 ( $\Delta$ ura3) to obtain the strains W29 ( $\triangle$ Ylyhm2 AnYHM2) and W29 (AnYHM2), respectively.

\subsection{Bacterial expression and purification}

YlYhm2p and YlCtp1p were expressed at $37^{\circ} \mathrm{C}$ as inclusion bodies in 
the cytosol of E. coli M15 (pREP4) as described previously (Agrimi et al., 2012b; Fiermonte et al., 1993; Marobbio et al., 2008). Control cultures with the empty vector were processed in parallel. Inclusion bodies were purified on a sucrose density gradient and washed at $4{ }^{\circ} \mathrm{C}$, first with TE buffer (10 mM Tris- $\mathrm{HCl}$ and $1 \mathrm{mM}$ EDTA, $\mathrm{pH} 7.0)$, then twice with a buffer containing $3 \%$ Triton X-114 (w/v), 1 mM EDTA and $10 \mathrm{mM}$ PIPES pH 7.0, and finally once with TE buffer (Agrimi et al., 2012a; Di Noia et al., 2014). Proteins were analyzed by SDS-PAGE on $17.5 \%$ gels and stained with Coomassie Blue dye. Unlike YlYhm2p, YlCtp1p was not expressed. Purified YlYhm2p was solubilized in 1\% sarkosyl (w/v), $10 \mathrm{mM}$ Tris- $\mathrm{HCl} \mathrm{pH} 7.0$ and $1 \mathrm{mM}$ EDTA, and unsolubilized material was removed by centrifugation $(15300 \times g$ for $10 \mathrm{~min}$ ). The identity of YlYhm2p which has been expressed in E. coli and purified was assessed by matrix-assisted laser desorption/ionization-time-of-flight (MALDI-TOF) mass spectrometry of trypsin digests of the corresponding band excised from Coomassie-stained gels (Hoyos et al., 2003; Palmieri et al., 2001).

The amount of purified YlYhm2p protein was estimated by laser densitometry of stained samples using carbonic anhydrase as protein standard (Indiveri et al., 1998; Monne et al., 2015).

\subsection{Reconstitution of YlYhm2p into liposomes and transport measurements}

The solubilized recombinant protein was diluted 3-fold with a buffer containing $3 \%$ Triton X-114 (w/v), $20 \mathrm{mM} \mathrm{Na}_{2} \mathrm{SO}_{4}$ and $10 \mathrm{mM}$ PIPES, $\mathrm{pH}$ 7.0, and reconstituted into liposomes by cyclic removal of the detergent with a hydrophobic column of amberlite beads (Bio-Rad) as previously described (Monné et al., 2018; Palmieri et al., 1995) with some modifications. The initial reconstitution mixture contained the solubilized protein (about $9 \mu \mathrm{g}$ ), 1\% Triton X-114, 1.4\% egg yolk phospholipids in the form of sonicated liposomes, $20 \mathrm{mM}$ substrate (except where otherwise indicated), $20 \mathrm{mM}$ PIPES pH 7.0, $0.6 \mathrm{mg}$ cardiolipin and water to a final volume of $700 \mu \mathrm{l}$ (Bisaccia and Palmieri, 1984). These components were mixed thoroughly, and the mixture was recycled 13 times through the amberlite column pre-equilibrated with a buffer containing $10 \mathrm{mM}$ PIPES ( $\mathrm{pH} \mathrm{7.0)}$ ) and the substrate at the same concentration used in the starting mixture. All operations were performed at $4{ }^{\circ} \mathrm{C}$, except for the passages through Amberlite, which were carried out at room temperature. The amount of purified protein incorporated into liposomes was measured as described (Porcelli et al., 2014 ) and was about $12 \%$ of protein added to the reconstitution mixture.

External substrate was removed from proteoliposomes on Sephadex G-75 columns pre-equilibrated with buffer A (10 mM PIPES and $50 \mathrm{mM}$ $\mathrm{NaCl} \mathrm{pH} \mathrm{7.0).} \mathrm{Transport} \mathrm{at} 25{ }^{\circ} \mathrm{C}$ was initiated by adding $\left[{ }^{14} \mathrm{C}\right]$ citrate (NEN Life Science Products) to eluted proteoliposomes. The reaction was terminated by adding $0.2 \mathrm{mM}$ p-hydroxymercuribenzoate which inhibits the activity of some MCs completely and rapidly (Marobbio et al., 2003, 2006). In controls, the inhibitor was added simultaneously to the labeled substrate according to the "inhibitor-stop" method (Palmieri et al., 1995). Finally, the external radioactivity was removed on Sephadex G-75 and the radioactivity in the proteoliposomes was measured. The experimental values were corrected by subtracting the control values. The initial transport rates were calculated from the radioactivity incorporated into proteoliposomes after $45 \mathrm{~s}$ (i. e. in the initial linear range of substrate transport) (Palmieri et al., 1999a). The kinetic constants $\mathrm{Km}$, Vmax and Ki were determined from LineweaverBurk and Dixon plots.

\subsection{Reconstitution of mitochondrial extract into liposomes and transport assays}

About $0.1 \mathrm{mg}$ protein of isolated mitochondria were solubilized with $1 \%$ Triton $\mathrm{X}-100,100 \mathrm{mM} \mathrm{NaCl}$ and $10 \mathrm{mM}$ PIPES, pH 7.0. After incubation for $20 \mathrm{~min}$ at $4^{\circ} \mathrm{C}$, the mixture was centrifuged at $138000 \times \mathrm{g}$ for $20 \mathrm{~min}$. The mitochondrial extract was reconstituted as described previously (Palmieri et al., 1999b). Transport was started by adding $\left[{ }^{14} \mathrm{C}\right]$ citrate, $\left[{ }^{14} \mathrm{C}\right] \alpha$-ketoglutarate (NEN Life Science Products) or $\left[{ }^{14} \mathrm{C}\right] \mathrm{ADP}$ (PerkinElmer) to proteoliposomes pre-loaded with $20 \mathrm{mM}$ of citrate, $\alpha$-ketoglutarate or ADP (as indicated in the legend to the figure) and terminated by the addition of $0.2 \mathrm{mM}$ p-hydroxymercurybenzoate. In controls, the inhibitor was added with the labeled substrate according to the "inhibitor-stop" method (Palmieri et al., 1995). The external radioactive substrate was removed, and the radioactivity in the proteoliposomes was measured as described previously (Palmieri et al., 1995). The experimental values were corrected by subtracting control values.

\subsection{Bioreactor fermentations}

Fermenter experiments were carried out in 1-L bioreactor (Applikon BioBundle $1 \mathrm{~L}$ ) with $0.5 \mathrm{~L}$ of medium containing $(\mathrm{g} / \mathrm{L}):\left(\mathrm{NH}_{4}\right)_{2} \mathrm{SO}_{4} 3.0$, $\mathrm{MgSO}_{4} \cdot 7 \mathrm{H}_{2} \mathrm{O} 1.4, \mathrm{NaCl} 0.5, \mathrm{Ca}\left(\mathrm{NO}_{3}\right)_{2} 0.8, \mathrm{KH}_{2} \mathrm{PO}_{4} 2.0, \mathrm{~K}_{2} \mathrm{HPO}_{4} 0.2$, biotin 0.0005 , thiamin 0.01 . Initial concentration of glucose was $40 \mathrm{~g}$ / L. Glucose was pulsed into bioreactor every $24 \mathrm{~h}$ at concentration of $30 \mathrm{~g} / \mathrm{L}$. Trace element stock solution has the following composition $(\mathrm{g} /$ L): $\mathrm{CuSO}_{4} \cdot 5 \mathrm{H}_{2} \mathrm{O}$ 6.0, KI 0.088, $\mathrm{MnSO}_{4} \cdot 5 \mathrm{H} 2 \mathrm{O}$ 3.0, $\mathrm{H}_{3} \mathrm{BO}_{3} \quad 0.2$, $\mathrm{CoCl}_{2} \cdot 6 \mathrm{H}_{2} \mathrm{O} \quad 0.955, \mathrm{ZnSO}_{4} \cdot 7 \mathrm{H}_{2} \mathrm{O}$ 42.0, $\mathrm{FeSO}_{4} \cdot 7 \mathrm{H}_{2} \mathrm{O}$ 65.0, $\mathrm{H}_{2} \mathrm{SO}_{4} 5.0$. $4.6 \mathrm{~mL}$ of the trace element stock solution was added to $1 \mathrm{~L}$ of medium. The temperature was maintained at the level of $29.0^{\circ} \mathrm{C}$, the concentration of dissolved oxygen was maintained at the level of $50-55 \%$. The $\mathrm{pH}$ was maintained at the level of 6.0. The selectivity of the process was calculated as the ratio of the weight of the target product (citric acid) to the weight of the products obtained (citric acid and isocitric acid).

\subsection{Analytical methods}

To determine the concentration of sugars and organic acids in the culture broth, cells were first precipitated by centrifugation at $8000 \times g$ for $3 \mathrm{~min}$, and then the supernatant was analyzed by high-performance liquid chromatography in $10 \mathrm{mM}$ aqueous solution of phosphoric acid, using a Waters HPLC system (Waters, Milford, MA, USA) fitted with a reversed-phase YMC-Triart C18 column $(4.6 \times 250 \mathrm{~mm}, 5 \mu \mathrm{m}, 12 \mathrm{~nm}$, YMC Co., Japan), and operating at $1.0 \mathrm{~mL} / \mathrm{min}$. Detection was performed at $210 \mathrm{~nm}$. Samples were identified by comparing the retention times with those of standards.

Lipids from aliquots of lyophilized cells $(10-20 \mathrm{mg}$ ) were extracted using the procedure of Folch et al. (1957). Gas chromatography was performed on a Shimadzu GC-2010 Plus instrument equipped with a flame-ionization detector and an SGE BPX 70 GC capillary column $(30 \mathrm{~m} \times 0.25 \mathrm{~mm} \times 0.25 \mu \mathrm{m})$. The GC oven conditions were as follows: $100{ }^{\circ} \mathrm{C}$ for $1 \mathrm{~min}$, ramped to $220^{\circ} \mathrm{C}$ in $15 \mathrm{~min}$, and held at $220^{\circ} \mathrm{C}$ for $2 \mathrm{~min}$. Heptadecanoic acid (C17:0, $50 \mu \mathrm{g}$ ) (Sigma-Aldrich, USA) was added as internal standard to a measured quantity of cell biomass. Fatty acids were quantified by comparison to commercial fatty acid methyl ester standards and normalized to methyl heptadecanoate (C17:0).

\section{Results}

\subsection{The identification of the main citrate transporter in Y. lipolytica mitochondria}

The Y. lipolytica genome harbors 39 genes coding for MC family type carrier proteins, in comparison to Homo sapiens (53 genes), Saccharomyces cerevisiae (35 genes) and Arabidopsis thaliana (58 genes) (Palmieri and Pierri, 2010). The sequences of the mitochondrial citrate carriers from S. cerevisiae (Yhm2p and Ctp1p) were used to find candidates for mitochondrial carrier(s) for citrate in $Y$. lipolytica. The closest relatives of Yhm2p and Ctp1p are two proteins of 311 and 292 amino acids, encoded by YALIOB10736g and YALIOF26323g, respectively, and named YlYhm2p and YlCtp1p for their high percentage of 
identical amino acids with ScYhm2p (76.9\%) and ScCtp1p (67.6\%). The sequences of YlYhm2p and YlCtp1p and their alignments (Fig. 1) with the closest relatives from $S$. cerevisiae and with the ADP/ATP carrier (the best studied member of the MC family) show that the proteins possess the distinct features of all members of the MC family. However, they share only $20 \%$ identical amino acids, a value very similar to the basic homology existing between the different members of the MC family, suggesting that YlYhm2p and YlCtp1p belong to two completely different subfamilies of MCs. A phylogenetic tree of all MCs in Y. lipolytica and S. cerevisiae is presented in Supplementary Fig. S4.

Deletion of the genes coding YlCtp1p and YlYhm2p was performed in $Y$. lipolytica yeast in order to discover the mitochondrial carrier playing a main role in citrate transport through the mitochondrial inner membrane of $Y$. lipolytica. In liquid glucose-containing minimal

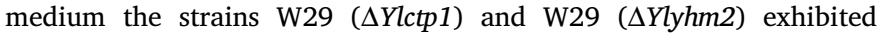
growth characteristics similar to those of the wild type strain W29 (Fig. 2A). Interestingly, in liquid citrate-containing minimal medium W29 $(\Delta$ Ylyhm2) failed to grow. In contrast, W29 ( $\Delta$ Ylctp1) grew to the same optical density as W29 (Fig. 2B), although this null mutant strain had an extended lag phase and a slightly lower specific growth rate $(\mu)$ compared to that of W29 $\left(0.20 \mathrm{~h}^{-1}\right.$ and $0.24 \mathrm{~h}^{-1}$, respectively) (Fig. 2B). Based on the growth curves of the deleted strains it can be assumed that catabolism of citrate occurs in mitochondria via TCA cycle and YlYhm2p is responsible for citrate import from the cytosol.

To investigate the influence of the introduced mutations on organic acid production and de novo lipid synthesis by $Y$. lipolytica the test tube cultivation was performed in the medium with excess of glucose and nitrogen starvation. The strain W29 ( $\triangle$ Ylctp1) did not differ significantly from the parental strain W29 in terms of biomass accumulation, organic acid production and lipid synthesis (Table 1). On the contrary, under the same conditions the strain W29 ( YYlyhm2) did not produce citric acid in the culture broth and did not utilize all glucose from the culture broth, despite the fact that there was no effect on final biomass accumulation compared to the wild type strain (Table 1). Total lipid content of W29 ( $\triangle$ Ylyhm2) was decreased to $6.8 \%$ of dry cell weight compared to $21.1 \%$ and $20.5 \%$ of dry cell weight observed for W29 ( $\Delta$ Ylctp1) and W29, respectively (Table 1). Furthermore, the level of isocitric acid production by the strain W29 ( $\triangle$ Ylyhm2) was decreased by approximately $60 \%$ compared to the wild type strain, and $\alpha$-ketoglutaric and malic acids accumulated in the culture broth (Table 1). These data show that the function of YlYhm2p is crucial in Y. lipolytica during growth on glucose-containing lipogenic medium.

To check whether the differences in growth, citric acid production and lipid accumulation displayed by the W29 ( $\triangle$ Ylyhm2) strain were the results of the absence of YlYhm2p and not a secondary effect, homologous expression of YlYHM2 gene in W29 ( $\mathrm{Ylyhm2}$ ) strain was evaluated. For this purpose YlYHM2 gene was cloned under strong constitutive intron-containing translation elongation factor- $1 \alpha$ promoter (TEFin) (Tai and Stephanopoulos, 2013) in a single-copy integrative vector. Indeed, the constructed strain W29 (AYlyhm2 YlYHM2) grew well on liquid citrate-containing minimal medium although the two replicates shown in Fig. 2B exhibited a different lag phase, probably due to a difference in cell survival of the seed culture. Nevertheless, $\mu$ parameters for the two replicates were very similar ( 0.22 and $\left.0.21 \mathrm{~h}^{-1}\right)$ and comparable to that of the wild type W29 strain $\left(0.24 \mathrm{~h}^{-1}\right)$ (Fig. 2B). During cultivation in glucose-containing lipogenic medium W29 ( $\triangle$ Ylyhm2 YlYHM2) produced $43.7 \mathrm{~g} / \mathrm{L}$ of citric acid and accumulated lipids to $21.5 \%$ of dry cell weight, demonstrating that reintroduction of the YlYHM2 gene in the deleted strain completely restores the lack of growth and citric acid production as well as the marked decrease in lipid accumulation (Table 1).

For a century, A. niger has been widely used for industrial production of citric acid (Papagianni, 2007). We evaluated whether the $A$. niger homolog to YlYhm2p had the same function and physiological role in citrate transporting. For this purpose AnYHM2 gene was over-expressed in W29 ( $\triangle$ Ylyhm2) and W29 strains. Indeed, the constructed strain W29 ( $\triangle$ Ylyhm2 AnYHM2) grew on liquid citrate-containing minimal medium. Despite an extended lag phase the specific growth rate of W29 ( $\triangle$ Ylyhm2 AnYHM2) was $0.22 \mathrm{~h}^{-1}$ compared with W29 being $0.24 \mathrm{~h}^{-1}$ (Fig. 2B). Moreover, W29 ( $\triangle$ Ylyhm2 AnYHM2) produced citric acid up to $43.6 \mathrm{~g} / \mathrm{L}$ (Table 1 ), that corresponded to an increase of $45 \%$ relative to wild type W29. AnYHM2 gene was also over-expressed in wild type strain W29. The strain W29 (AnYHM2) produced $37.3 \mathrm{~g} / \mathrm{L}$ of citric acid that corresponded to an increase of $24 \%$ relative to W29 (Table 1).

To understand the contribution of YlYhm2p to citrate and $\alpha$-ketoglutarate transport in $Y$. lipolytica mitochondria, these organelles

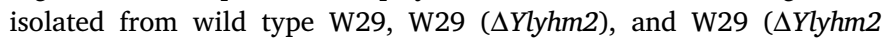
YlYHM2) cells were solubilized. Then the mitochondrial extracts were incorporated into liposomes, and the transport of citrate and $\alpha$-ketoglutarate, present either inside or outside the liposomes or on both sides, was tested. In liposomes reconstituted with the mitochondrial extract from W29 ( Ylyhm2) $\left[{ }^{14} \mathrm{C}\right]$ citrate/citrate and $\left[{ }^{14} \mathrm{C}\right] \alpha$-ketoglutarate/ $\alpha$-ketoglutarate homoexchanges were reduced by $87 \%$ and $40 \%$, respectively, $(\mathrm{P}<0.05)$, and the heteroexchanges citrate $_{\mathrm{in}} / \alpha$-ketoglutarate ${ }_{\text {out }}$ and $\alpha$-ketoglutarate ${ }_{\text {in }} /$ citrate $_{\text {out }}$ were diminished by $87 \%$ and $95 \%$, respectively, as compared with the activities of the wild type strain $(\mathrm{P}<0.05)$ (Fig. 3). As a control, the $\left[{ }^{14} \mathrm{C}\right] \mathrm{ADP} / \mathrm{ADP}$ homoexchange was also measured and found to be comparable in the mitochondrial extracts from W29, W29 ( $\triangle$ Ylyhm2), and W29 ( $\triangle$ Ylyhm2 YlYHM2) strains (Supplementary Fig. S5). Thus, the deletion of YlYHM2 caused a dramatic decrease in citrate transport and a less pronounced,

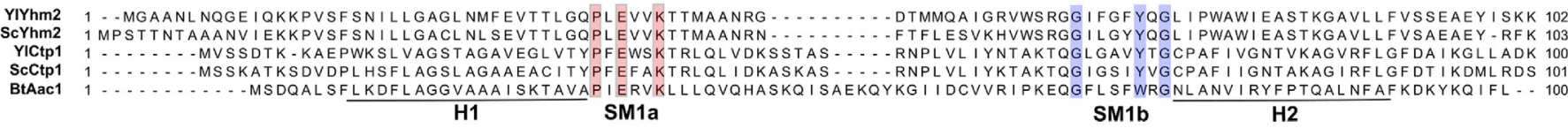

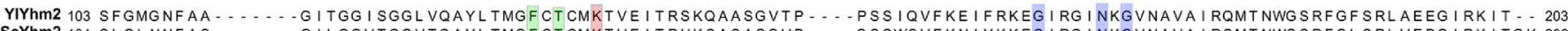

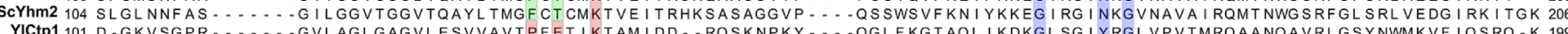
YICtp1 101 D - G KVSGPR - - . . . - GVLAGLGAGVLESVVAVTPFET I KT AM I DD - RQSKNPKY - - - Q QGLFKGTAQL I KDKGLSG I YRGLVPVTMRQAANQAVRLGSYNWMKVF IQSRQ - K 199

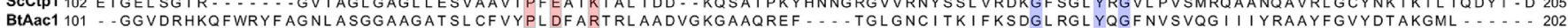
H3 SM2a SM2b H4

YIYhm2 204 KNDDRPLNAFEK I AASTVGGGL SAWNQ - P I EV IRVEMQSKTVDPNRPK - NL SVWTAFKY I YQQNGIKGLYRGVTPRIGLGIWQTVCMVGLGDIAKDWVAKVTGERPVGKH ScYhm2 207 TNKDDKLNPFEK I GASALGGGL SAWNQ - PI EV I RVEMQSKKEDPNRPK - NL TVGKTFKY I YQSNGL KGL YRGVTPR I GLG I WQTVFMVGFGDMAKEFVARMTGETPVAKH YICtp1 200 DPKA - PLSSLSTF I VGAFAG I VTVYTTMPLDTVKTRMQSLEAKKE - . - YRGTFHCFARIFKEEGLLTFWKGATPRLGRL ILSGGIVFT IYEK IME IL -

ScCtp1 206 SPKDKPLSSGLTFLVGAFSG IVTVYSTMPLDTVKTRMQSLDSTK . . . . YSSTMNCFAT IFKEEGLKTFWKGATPRLGRLVLSGGIVFTIYEKVLVMLA .

BtAac1 203 - PDPKNVHI IVSWMI AQTVTAVAGLVSYPFDTVRRRMMMQSGRKGADIMYTGTVDCWRKIAKDEGPKAFFKGAWSNVLR - GMGGAFVLVLYDEIKKFV . . . . . . . . . H5 SM3a

SM3b

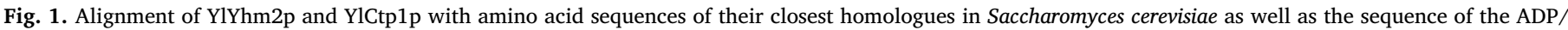

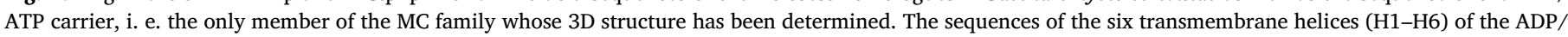

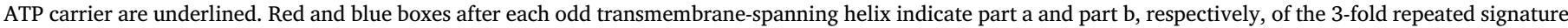

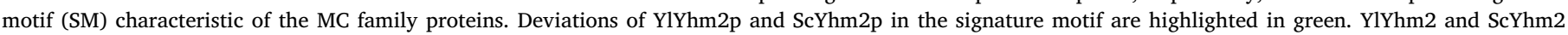

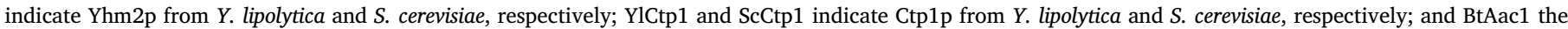
$\mathrm{ADP} / \mathrm{ATP}$ carrier from Bos taurus. (For interpretation of the references to colour in this figure legend, the reader is referred to the Web version of this article.) 

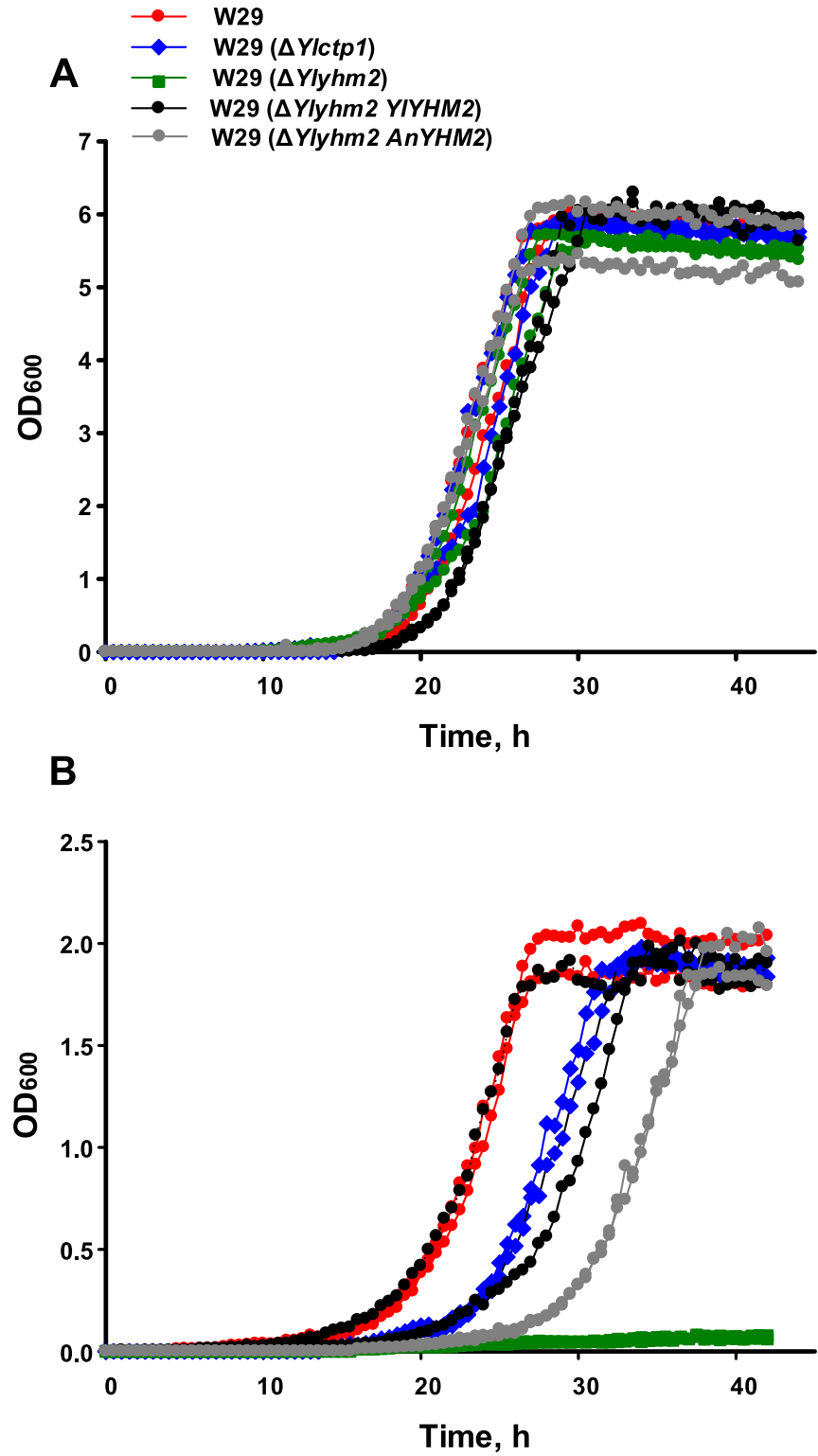

Fig. 2. Growth curves of $Y$. lipolytica strains W29 (red), W29 ( $\triangle$ Ylctp1) (blue),

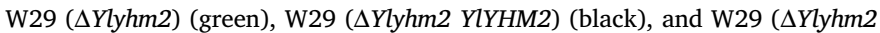
AnYHM2) (grey) grown on minimal medium containing glucose (A) and citrate (B). All replicates of strains were inoculated to a final titre of $3 \times 10^{5}$ cells $/ \mathrm{mL}$ counting with the hemocytometer. (For interpretation of the references to colour in this figure legend, the reader is referred to the Web version of this article.)

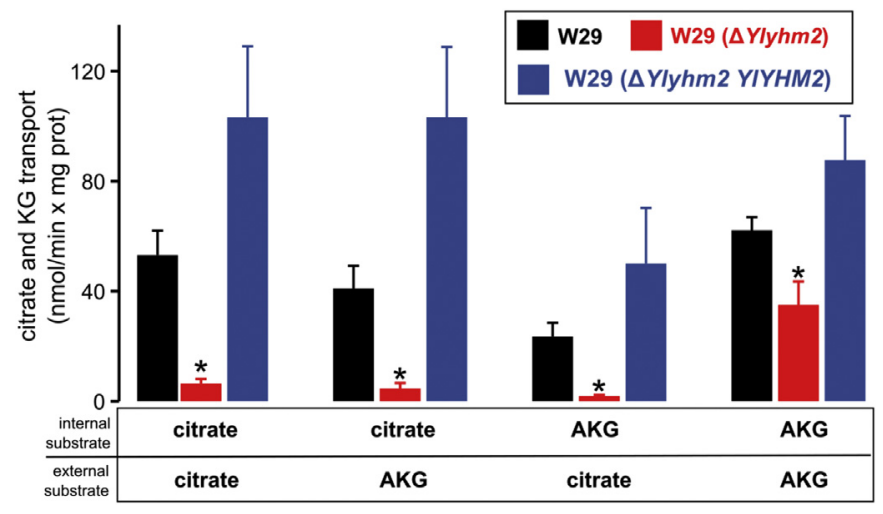

Fig. 3. Citrate and $\alpha$-ketoglutarate homoexchange and heteroexchange activities in liposomes reconstituted with mitochondrial extracts of wild type W29, W29 (AYlyhm2), and W29 ( Ylyhm2 YlYHM2) cells. The extract ( $25 \mu \mathrm{g}$ of protein) of isolated mitochondria from wild type W29 (black columns), W29 ( $\triangle$ Ylyhm2) (red columns), or W29 ( $\triangle$ Ylyhm2 YlYHM2) (blue columns) strains was reconstituted into liposomes preloaded with $20 \mathrm{mM} \alpha$-ketoglutarate or citrate. Transport was initiated by adding $1.2 \mathrm{mM}\left[{ }^{14} \mathrm{C}\right] \alpha$-ketoglutarate or $0.15 \mathrm{mM}\left[{ }^{14} \mathrm{C}\right]$ citrate to proteoliposomes and terminated after $5 \mathrm{~min}$. The data represent the mean \pm SEM for at least three independent experiments performed in duplicate. ": significant difference of the mutant strain transport activity as compared to the wild type strain $(\mathrm{P}<0.05 t$-test). (For interpretation of the references to colour in this figure legend, the reader is referred to the Web version of this article.)

but substantial, decrease in $\alpha$-ketoglutarate transport in isolated mitochondria of $Y$. lipolytica. Furthermore, all the exchanges investigated involving either citrate or $\alpha$-ketoglutarate were more than fully restored by reintroducing the YlYHM2 gene in the deleted strain (Fig. 3). Therefore, in Yarrowia mitochondria YlYhm2p is virtually the sole citrate transporter and one of the main, but not the sole, $\alpha$-ketoglutarate transporter.

\subsection{The characteristics of recombinant YlYhm2p}

Reconstitution of recombinant proteins in liposomes is a commonly used method to identify and characterize the transport properties of carrier proteins encoded by different genomes (Palmieri and Monné, 2016). Therefore, the YlYHM2 and YlCTP1 genes were expressed in E. coli M15 (pREP4) cells. YlCTP1 failed to be expressed most likely because it is toxic to the bacterial cells. In contrast, the YlYHM2 gene product accumulated as inclusion bodies and was purified by centrifugation and washing (Fig. 4A, lane 5). The apparent molecular mass of the purified protein was about $32.8 \mathrm{kDa}$, in good agreement with its calculated value $(33.7 \mathrm{kDa})$. The identity of the recombinant protein was confirmed by MALDI-TOF mass spectrometry and the yield of the

Table 1

Biomass accumulation, glucose consumption, organic acid production, and total lipid content of $Y$. lipolytica recombinant strains under cultivation for 5 days in minimal lipogenic medium with glucose as a sole carbon source. Values are reported as average \pm standard deviation from three independent experiments.

\begin{tabular}{|c|c|c|c|c|c|c|c|}
\hline \multirow[t]{2}{*}{ Strain } & \multirow[t]{2}{*}{ Biomass, $\mathrm{g} \mathrm{dcw} / \mathrm{L}$} & \multirow[t]{2}{*}{ Glucose consumed, g/L } & \multirow[t]{2}{*}{ Total fatty acids, $\% \mathrm{dcw}$} & \multicolumn{4}{|c|}{ Organic acids, g/L } \\
\hline & & & & Citric acid & Isocitric acid & $\alpha$-Ketoglutaric acid & Malic acid \\
\hline W29 ( $\Delta Y l c t p 1)$ & $16.7 \pm 3.4$ & $90.0 \pm 0.0$ & $21.1 \pm 1.6$ & $32.1 \pm 0.6$ & $11.2 \pm 0.4$ & $\mathrm{ND}^{\mathrm{a}}$ & ND \\
\hline W29 (AYlyhm2) & $17.0 \pm 2.0$ & $67.7 \pm 6.1$ & $6.8 \pm 2.8$ & $0.05 \pm 0.05$ & $3.4 \pm 1.4$ & $2.0 \pm 0.5$ & $2.3 \pm 0.3$ \\
\hline W29 (AYlyhm2 YlYHM2) & $16.3 \pm 1.6$ & $90.0 \pm 0.0$ & $21.5 \pm 1.1$ & $43.7 \pm 8.2$ & $3.5 \pm 0.9$ & ND & ND \\
\hline W29 (YlYHM2) & $16.1 \pm 3.9$ & $90.0 \pm 0.0$ & $20.9 \pm 0.8$ & $37.1 \pm 1.0$ & $4.0 \pm 0.3$ & ND & ND \\
\hline W29 ( $\triangle$ Ylyhm2 AnYHM2) & $17.0 \pm 1.4$ & $90.0 \pm 0.0$ & $19.9 \pm 1.3$ & $43.6 \pm 3.3$ & $4.2 \pm 0.4$ & ND & ND \\
\hline W29 (AnYHM2) & $14.3 \pm 2.1$ & $90.0 \pm 0.0$ & $19.4 \pm 1.3$ & $37.3 \pm 2.4$ & $4.4 \pm 0.2$ & ND & ND \\
\hline W29 (YLAMPD) & $16.5 \pm 2.5$ & $90.0 \pm 0.0$ & $17.7 \pm 2.2$ & $39.2 \pm 3.6$ & $8.3 \pm 1.2$ & ND & ND \\
\hline W29 (YlAMPD YlYHM2) & $16.0 \pm 3.0$ & $90.0 \pm 0.0$ & $16.9 \pm 1.5$ & $49.7 \pm 1.5$ & $4.4 \pm 0.2$ & ND & ND \\
\hline wild type W29 & $16.6 \pm 2.7$ & $90.0 \pm 0.0$ & $20.5 \pm 1.4$ & $30.1 \pm 2.7$ & $8.4 \pm 1.1$ & ND & ND \\
\hline
\end{tabular}

\footnotetext{
a $-\mathrm{ND}$, not detected.
} 


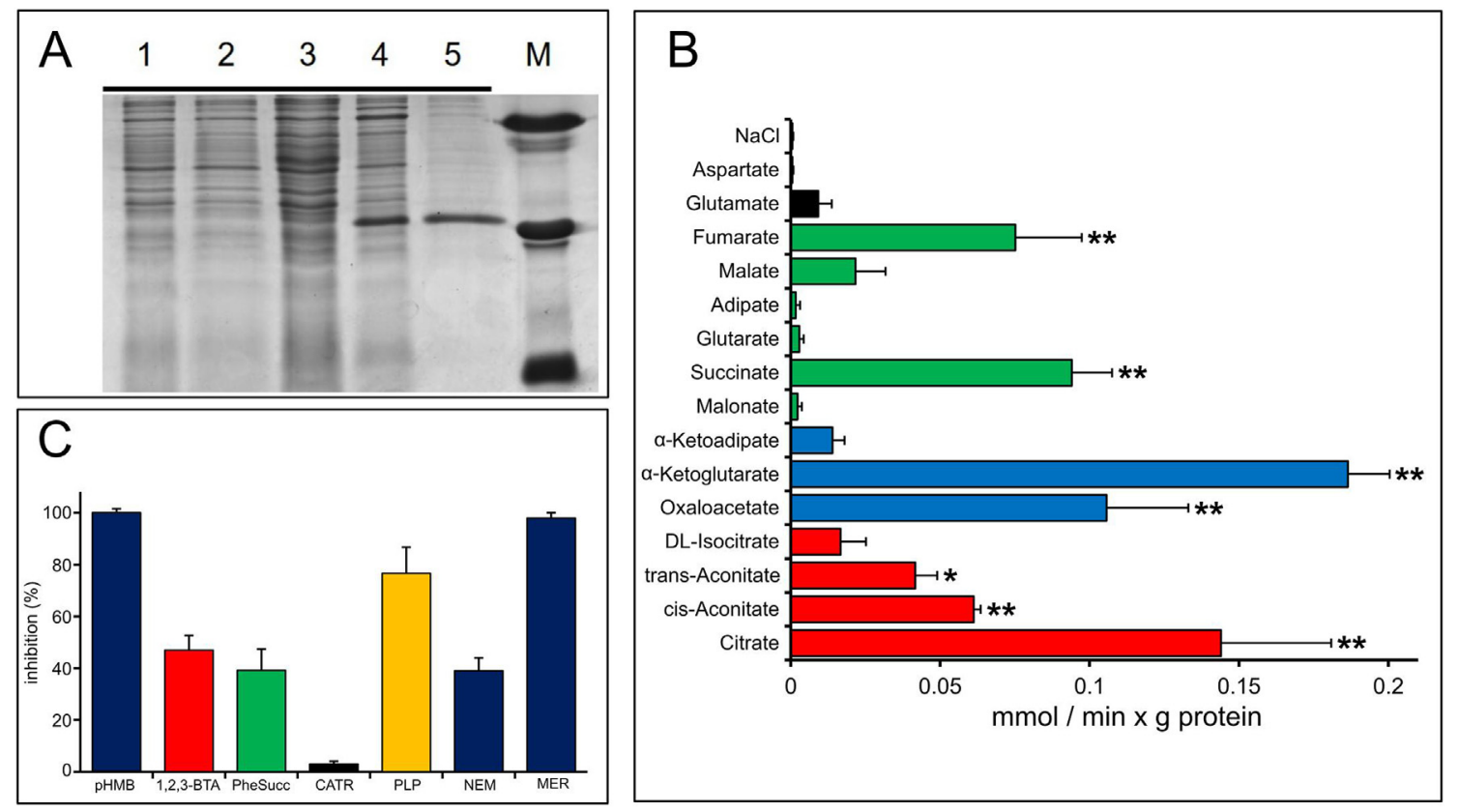

Fig. 4. Expression in E. coli and properties of recombinant YlYhm2p. (A) Expression of YlYhm2p in E. coli and its purification. Proteins were separated by SDS-PAGE and stained with Coomassie Blue dye. Markers (bovine serum albumin, carbonic anhydrase and cytochrome $c$ ) are shown in the right column. Lanes 1-4, E. coli M15 (pREP4) cells containing the expression vector with (lanes 2 and 4) and without (lanes 1 and 3) the coding sequence of YlYhm2p. Samples were taken immediately before (lanes 1 and 2) and $4 \mathrm{~h}$ after induction (lanes 3 and 4). The same number of bacteria was analyzed in each sample. Lane 5, purified YlYhm2p (about 10 $\mu \mathrm{g}$ ) derived from the bacteria shown in lane 4. (B) Dependence of YlYhm2p activity on internal substrate. Liposomes reconstituted with YlYhm2p were preloaded internally with various substrates (concentration, $20 \mathrm{mM}$ ). Transport was initiated by adding $0.15 \mathrm{mM}\left[{ }^{14} \mathrm{C}\right]$ citrate and terminated after $45 \mathrm{~s}$. The values are means \pm SEM of at least three independent experiments in duplicate for each internal substrate investigated. In red: tricarboxylic acids. In blue: $\alpha$-ketodicarboxylic acids. In green: dicarboxylic acids. In black: other compounds. Differences between the activities of citrate uptake with internal citrate, cis-aconitate, trans-aconitate, oxaloacetate, $\alpha$-ketoglutarate, succinate and fumarate and the activity with internal $\mathrm{NaCl}$ were significant (Fisher $t$-test $* \mathrm{p}<0.05$, **p $<0.01$ ). (C) Effect of inhibitors on the citrate/citrate exchange by YlYhm2p. Liposomes were reconstituted with YlYhm2p and preloaded internally with $20 \mathrm{mM}$ citrate. Transport was initiated by adding $0.15 \mathrm{mM}\left[{ }^{14} \mathrm{C}\right]$ citrate and terminated after $45 \mathrm{~s}$. The concentrations of the inhibitors were $10 \mu \mathrm{M}$ (CATR, carboxyatractyloside), $0.2 \mathrm{mM}$ (MER, mersalyl; pHMB, p-hydroxymercuribenzoate), $1 \mathrm{mM}$ (NEM, N-ethylmaleimide), $2 \mathrm{mM}$ (PheSucc, phenylsuccinate; 1,2,3-BTA, 1,2,3-benzenetricarboxylate), 30 mM (PLP, pyridoxal 5'-phosphate). The values are means \pm SEM of at least three independent experiments in duplicate for each inhibitor investigated. (For interpretation of the references to colour in this figure legend, the reader is referred to the Web version of this article.)

purified protein was about $15 \mathrm{mg}$ per liter of culture. Notably, YlYhm2p was not detected in bacteria harvested immediately before induction of expression (Fig. 4A, lane 2) nor in cells harvested after induction but lacking the coding sequence in the expression vector (Fig. 4A, lane 3).

In a first set of experiments, recombinant purified YlYhm2p was reconstituted into liposomes, and its transport activities for a variety of potential substrates were tested in homoexchange experiments (i. e. with the same substrate inside and outside the proteoliposomes). Using external and internal substrate concentrations of $50 \mu \mathrm{M}$ and $10 \mathrm{mM}$, respectively, reconstituted YlYhm2p catalyzed active ${ }^{14}[\mathrm{C}]$ citrate/citrate and $\left[{ }^{14} \mathrm{C}\right] \alpha$-ketoglutarate/ $\alpha$-ketoglutarate homoexchanges, which were temperature dependent and inhibited by the SH-reagent p-hydroxymercuribenzoate (data not shown), as would be expected from a protein-mediated transport. In addition, no $\left[{ }^{14} \mathrm{C}\right]$ citrate/citrate and $\left[{ }^{14} \mathrm{C}\right] \alpha$-ketoglutarate/ $\alpha$-ketoglutarate exchange activities were detected when YlYhm2p was inactivated by boiling before reconstitution into liposomes or when liposomes were reconstituted with sarkosyl-solubilized protein from bacterial cells either lacking the expression vector or harvested immediately before induction of expression (data not shown).

To investigate the substrate specificity of YlYhm2p in detail, the initial rate of $0.15 \mathrm{mM}\left[{ }^{14} \mathrm{C}\right]$ citrate uptake into proteoliposomes, that had been preloaded with a variety of potential substrates, was measured (Fig. 4B). The highest activities of $\left[{ }^{14} \mathrm{C}\right]$ citrate uptake into proteoliposomes were observed with internal citrate, $\alpha$-ketoglutarate, oxaloacetate, succinate and fumarate. $\left[{ }^{14} \mathrm{C}\right]$ citrate also exchanged substantially with internal cis-aconitate and trans-aconitate and, to a lower extent, with isocitrate, oxoadipate and malate. In contrast, the YlYhm2p-mediated $\left[{ }^{14} \mathrm{C}\right]$ citrate uptake was negligible, or not significantly higher than that found in the presence of $\mathrm{NaCl}$ when the following internal substrates were used: malonate, glutarate, adipate, glutamate, aspartate, and $\mathrm{NaCl}$ and no substrate (Fig. 4B) as well as pyruvate, carnitine, $\alpha$-ketoisovalerate, glutamine, methionine, cysteine, alanine, valine, leucine, S-adenosylmethionine, glutathione, ADP, ATP, GDP, GTP, UDP, UTP, CDP, CTP, NAD ${ }^{+}$, FAD, thiamine pyrophosphate, and coenzyme A (data not shown). Therefore, the substrate specificity of YlYhm2p is mainly confined to tricarboxylic acids (mainly citrate), C4- and C5-oxodicarboxylic acids, and to the C4-dicarboxylates succinate and fumarate.

The effects of MC inhibitors on the $\left[{ }^{14} \mathrm{C}\right]$ citrate/citrate exchange reaction catalyzed by reconstituted YlYhm2p were also examined (Fig. 4C). This activity was inhibited strongly by the SH-blocking reagents, such as p-hydroxymercurybenzoate and mersalyl, by the lysine $\varepsilon$-amino group reagent pyridoxal-5'-phosphate, and partially by the alkylating reagent $\mathrm{N}$-ethylmaleimide. $\left[{ }^{14} \mathrm{C}\right]$ Citrate/citrate exchange was also inhibited by $2 \mathrm{mM}$ 1,2,3-benzenetricarboxylate (an inhibitor of the citrate carrier (Palmieri et al., 1972)) and $2 \mathrm{mM}$ phenylsuccinate (an inhibitor of the dicarboxylate carrier (Palmieri et al., 1971)). In contrast, carboxyatractyloside, a potent inhibitor of the ADP/ATP carrier (Klingenberg, 2008) had very little effect on YlYhm2p activity.

The kinetic constants of the reconstituted YlYhm2p were determined by measuring the initial transport rate of the $\left[{ }^{14} \mathrm{C}\right]$ citrate/ citrate exchange in the presence of various external $\left[{ }^{14} \mathrm{C}\right]$ citrate concentrations and a constant saturating internal substrate concentration of citrate $(20 \mathrm{mM})$. The transport affinity $(\mathrm{Km})$ and the specific activity (Vmax) values for the citrate/citrate exchange at $25^{\circ} \mathrm{C}$ were $0.15 \pm 0.01 \mathrm{mM}$ and $0.34 \pm 0.06 \mathrm{mmol} / \mathrm{min}$ per $\mathrm{g}$ of protein, 
respectively, in 15 experiments.

Several external substrates were competitive inhibitors of $\left[{ }^{14} \mathrm{C}\right] \mathrm{ci}$ trate uptake as they increased the apparent $\mathrm{Km}$ of the citrate/citrate exchange without changing the Vmax (results not shown). The Ki values of these substrates for YlYhm2p were as follows: $1.1 \mathrm{mM} \pm 0.12$ ( $\alpha$-ketoglutarate); $0.33 \mathrm{mM} \pm 0.01$ (oxaloacetate); $0.55 \mathrm{mM} \pm 0.02$ (succinate); $0.76 \mathrm{mM} \pm 0.08$ (fumarate).

\subsection{Effect of YlYHM2 gene over-expression on citric acid production}

YlYHM2 gene was over-expressed in wild type W29 and W29 (YLAMPD) strains, the latter carrying an additional copy of YLAMPD gene expressed under hp4d promoter. The positive effect of YlAMPD gene over-expression to itaconic acid production has been previously demonstrated (Blazeck et al., 2015). Over-expression of YlYHM2 and YLAMPD genes separately increased citric acid production by approximately 24 and 31\%, respectively, compared with wild type (Table 1). At the same time, a decrease in lipid accumulation and isocitric acid production was observed for W29 (YLAMPD) and W29 (YlYHM2) strains, respectively. Thus, W29 (YlAMPD) accumulated lipids to $17.7 \%$ of dry cell weight, whereas lipid accumulation by W29 was $20.5 \%$ of dry cell weight. Production of isocitric acid by W29 (YlYHM2) strain was decreased by $50 \%$ compared with W29. Notably, co-expression of YlYHM2 and YlAMPD genes improved considerably citric acid production. Thus, W29 (YlAMPD YlYHM2) produced $49.7 \mathrm{~g} / \mathrm{L}$ of citric acid under test tube cultivation, i. e. $65 \%$ more than the wild type strain. Furthermore, under the same conditions lipid accumulation and isocitric acid production by W29 (YLAMPD YlYHM2) were decreased by $18 \%$ and $48 \%$, respectively, compared with W29.

Large-scale cultivation of W29 (YLAMPD YlYHM2) and W29 was conducted using 1-L stirred tank bioreactor (Fig. 5). In the phase of active cell growth (up to $24 \mathrm{~h}$ ) no marked citric acid excretion was observed for both strains (Fig. 5A). However, during the period from 24 to $120 \mathrm{~h}$ W29 (YLAMPD YlYHM2) strain produced intensively citric acid. In this period, the average specific rate of citric acid synthesis was $0.80 \mathrm{~g} / \mathrm{L} / \mathrm{h}$, and the maximal specific rate of $0.93 \mathrm{~g} / \mathrm{L} / \mathrm{h}$ was reached from 76 to $96 \mathrm{~h}$ (Fig. 5A). The average specific rate of citric acid synthesis by the control strain W29 from 24 to $120 \mathrm{~h}$ was $0.61 \mathrm{~g} / \mathrm{L} / \mathrm{h}$; the maximum of $0.92 \mathrm{~g} / \mathrm{L} / \mathrm{h}$ was observed from 96 to $120 \mathrm{~h}$ (Fig. 5A). From 120 to $166 \mathrm{~h}$ both strains continued to produce citric acid but the specific rates of citric acid synthesis decreased approximately twofold and threefold for W29 (YlAMPD YlYHM2) and W29 strains, respectively, as compared with their maximum values. By $166 \mathrm{~h}$ of cultivation, W29 (YlAMPD YlYHM2) accumulated $97.1 \mathrm{~g} / \mathrm{L}$ of citric acid and $6.0 \mathrm{~g} / \mathrm{L}$ of isocitric acid with a process selectivity of $94.2 \%$ and an overall citric acid yield of $0.5 \mathrm{~g} / \mathrm{g}$. At the same time of cultivation the culture broth of W29 contained $73.2 \mathrm{~g} / \mathrm{L}$ of citric acid and $8.5 \mathrm{~g} / \mathrm{L}$ of isocitric acid that corresponds to a process selectivity of $89.6 \%$ and an overall citric acid yield of $0.38 \mathrm{~g} / \mathrm{g}$.

\section{Discussion}

During carbohydrate utilization, the majority of eukaryotes use citrate, generated in the mitochondrial matrix by the TCA cycle, to supply acetyl-CoA in the cytoplasm for the synthesis of important compounds such as fatty acids, sterols and $\mathrm{N}$-acetylglucosamine. The translocation of citrate across the mitochondrial membrane, catalyzed by the mitochondrial citrate carrier, is an essential metabolic step in these processes.

$Y$. lipolytica belongs to the group of oleaginous microorganisms that have the potential to accumulate a substantial amount of lipids (Beopoulos et al., 2011). At the same time, Y. lipolytica is able to secrete citric and isocitric acids (Cavallo et al., 2017; Kamzolova et al., 2018). These properties clearly show that $Y$. lipolytica can serve as a model yeast to evaluate the mechanisms of acetyl-unit transfer from the mitochondria to the cytosol. The aim of this study was the identification and functional characterization of the transport protein(s) responsible for citrate translocation across the $Y$. lipolytica mitochondrial membrane and therefore essential for the biosynthetic potentials of this organism. The results presented here clearly demonstrate that YlYhm2p is essential for the import of citrate into mitochondria when citrate is used as a single carbon source (Fig. 2B). Our data also show that YlYhm2p plays an essential role in the export of citrate from mitochondria on glucose-containing lipogenic medium. This conclusion is substantiated by the fact that secretion of citric acid is abolished and the production of isocitric acid and lipids is severely decreased in the W29 ( $\triangle$ Ylyhm2) strain during cultivation under nitrogen starvation with excess of glucose (Table 1). Moreover, direct transport measurements in reconstituted mitochondrial extracts of W29 ( $\triangle$ Ylyhm2) and wild type strains demonstrate that the activity of citrate transport is almost absent in the mitochondrial extracts of the deleted strain, whereas that of ADP transport is virtually the same (Fig. 3 and Supplementary Fig. S5). In addition, reintroduction of YlYHM2 in the W29 (AYlyhm2) strain fully restores growth in liquid citrate-containing minimal medium as well as citric acid production and lipid accumulation in glucose-containing lipogenic medium (Fig. 2B, Table 1). Altogether, the above results provide strong evidence that $\mathrm{YlYhm} 2 \mathrm{p}$ is the primary mitochondrial citrate

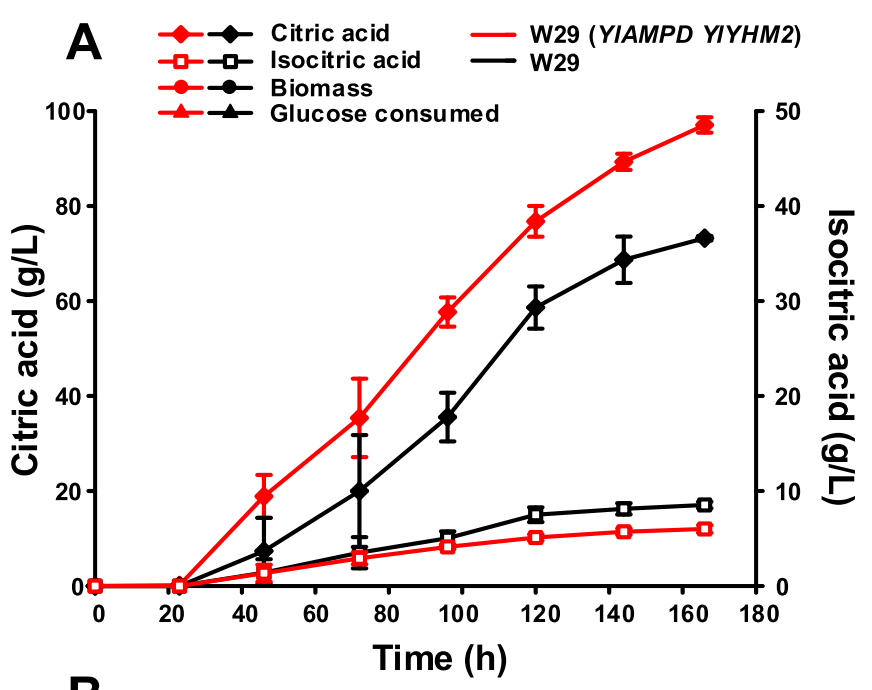

B

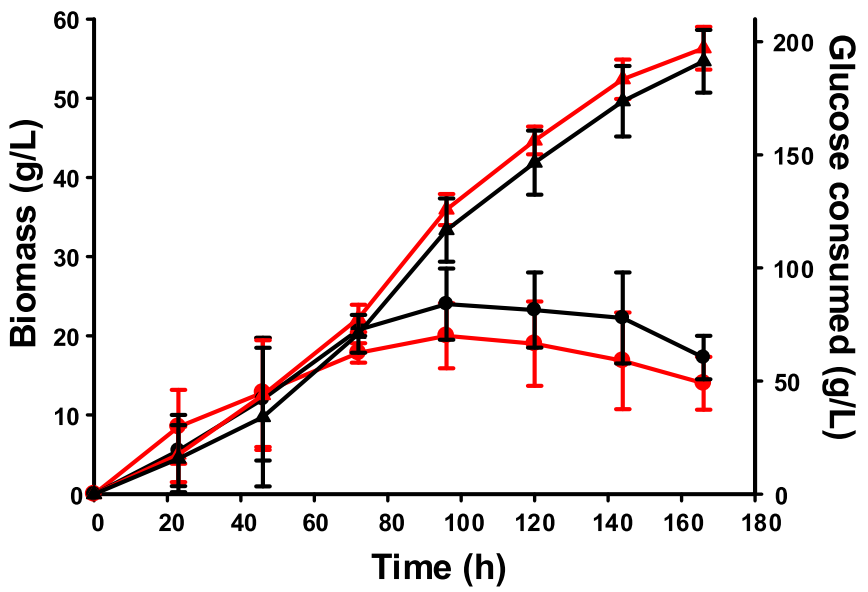

Fig. 5. Batch bioreactor performance of W29 (YlAMPD YlYHM2) and W29 strains with glucose pulse-feeding. Glucose was added every $24 \mathrm{~h}$ at a concentration of $30 \mathrm{~g} / \mathrm{L}$. Citric and isocitric acid concentrations are reported in panel (A) and biomass and glucose consumed in panel (B). Error bars indicate SEM calculated from three independent experiments. (For interpretation of the references to colour in this figure legend, the reader is referred to the Web version of this article.) 
carrier in Y. lipolytica. Moreover, it was demonstrated that A. niger AnYhm2p possessed the function of citrate transporting in $Y$. lipolytica and could substitute YlYhm2p (Table 1). In the very recently published article, AnYhm2p was also identified and studied in A. niger (Kirimura et al., 2019). However, the deletion of the AnYHM2 gene in A. niger reduces, but not abolishes, citric acid production (Kirimura et al., 2019), indicating that another mitochondrial carrier for citrate exists in A. niger and the over-expression of AnYHM2 had not been carried out until now.

Here we have also found that inactivation of YlYHM2 in Y. lipolytica does not prevent the growth on the minimal medium with glucose (Fig. 2A) nor de novo synthesis of neutral lipids although the latter is considerably lowered (Table 1). It should be noted that Y. lipolytica strain with inactivated ACL1 gene coding the subunit of ATP-dependent citrate lyase had also been reported to be able to grow on glucosecontaining media in spite of a clear delay in growth (Dulermo et al., 2015). These observations suggest that there is an alternative source of acetyl-CoA on carbohydrate substrates in $Y$. lipolytica.

The transport properties and kinetic parameters of recombinant and reconstituted YlYhm2p show that this protein transports citrate and $\alpha$ ketoglutarate with high efficiency, and oxaloacetate, succinate, and fumarate to a lesser extent, by a fast counter-exchange mode (Fig. 4B). In $S$. cerevisiae $Y h m 2 p$ is responsible for the citrate/ $\alpha$-ketoglutarate NADPH redox shuttle that transports reducing equivalents from the mitochondrial matrix to the cytosol via the Yhm2p-mediated exchange of intramitochondrial citrate for cytosolic $\alpha$-ketoglutarate (Castegna et al., 2010). In this system, citrate transported from the mitochondria is converted to $\alpha$-ketoglutarate by the action of cytosolic $\mathrm{NADP}^{+}$-dependent isocitrate dehydrogenase, and the resulting $\alpha$-ketoglutarate is shuttled back to the mitochondria to complete the cycle. However, in $Y$. lipolytica there is only one $\mathrm{NADP}^{+}$-dependent isocitrate dehydrogenase that is localized in the mitochondria (Li et al., 2013). In fact, the main source of reduced NADPH for anabolic and antioxidant reactions in $Y$. lipolytica is the pentose phosphate pathway (Wasylenko et al., 2015; Yuzbashev et al., 2016). We think that in Y. lipolytica growing on glucose, YlYhm2p catalyses the export of citrate mainly in exchange for cytosolic oxaloacetate (Fig. 6A). This proposal is supported by the following evidence: i) oxaloacetic acid is a good substrate for YlYhm2p (Fig. 4B), ii) under our experimental conditions oxaloacetic acid can be produced from pyruvate in the cytoplasm by the activity of pyruvate carboxylase, and iii) oxaloacetic acid is also accepted by $S$. cerevisiae
Yhm2p (Castegna et al., 2010). Other good counter-substrates for YlYhm2p are succinate, fumarate and, to a much lower extent, malate. We also envisaged the possibility that the export of citrate from the mitochondrial matrix involves a citrate $\mathrm{in}_{\text {in }} / \alpha$-ketoglutarate ${ }_{\text {out }}$ exchange, catalyzed by YlYhm2p, and an $\alpha$-ketoglutarate out $_{\text {t }} /$ alate $_{\text {out }}$ exchange mediated by the oxodicarboxylate carrier (Odc) of Y. lipolytica (Fig. 6B). To verify this hypothesis we inactivated YlODC1 gene (YALIOD02629g) in $Y$. lipolytica. $Y$. lipolytica contains only one $O D C$ gene unlike $S$. cerevisiae (Palmieri et al., 2001). We succeeded to perform the inactivation of YlODC1 only in YlKU7O null mutant strain (Supplementary Methods) in which the mechanism of non-homologous end-joining is hampered and, consequently, the efficiency of integration by homologous recombination is increased (Kretzschmar et al., 2013; Verbeke et al., 2013). However, the strain with inactivated YlODC1 gene accumulated approximately the same amount of citrate as the control strain (Supplementary Table S2). In this respect, it is worth mentioning that the possibility that another not yet identified MC for $\alpha$-ketoglutarate/ malate exchange exists in $Y$. lipolytica to complete the suggested citrate/ $\alpha$-ketoglutarate shuttle (Fig. 6B) cannot be excluded.

Metabolic engineering strategies aiming at improving the production of a target metabolite are often based on the manipulation of enzyme levels in the cell. In this study, to the best of our knowledge, for the first time the increased production of a metabolite synthesized in the mitochondria has been achieved by over-expressing the gene encoding its mitochondrial transporter. Thus, the new strain W29 (YlAMPD YlYHM2), over-expressing YlYHM2 and adenosine monophosphate deaminase (YLAMPD), produces high amounts of citric acid (up to $97.1 \mathrm{~g} / \mathrm{L}$ ) with maximal specific rate of $0.93 \mathrm{~g} / \mathrm{L} / \mathrm{h}$ and a process selectivity of $94.2 \%$. Very recently, the major plasma membrane transporter for citrate, named CexA, has been identified and examined in A. niger (Steiger et al., 2019). This information allows us to propose the over-expression of the $Y$. lipolytica homologous gene, $Y A$ LIOF03751g, or CexA together with YlYHM2 and YlAMPD to further increase the production of citric acid.

In conclusion, the data presented in this study highlight the importance of both YlYHM2 and AnYHM2 genes, and possibly their homologues as the target genes for genetic engineering manipulation to enhance citric acid production by mycelial fungi and yeasts, thereby opening new perspectives for improvement of industrial citric acid producing strains.

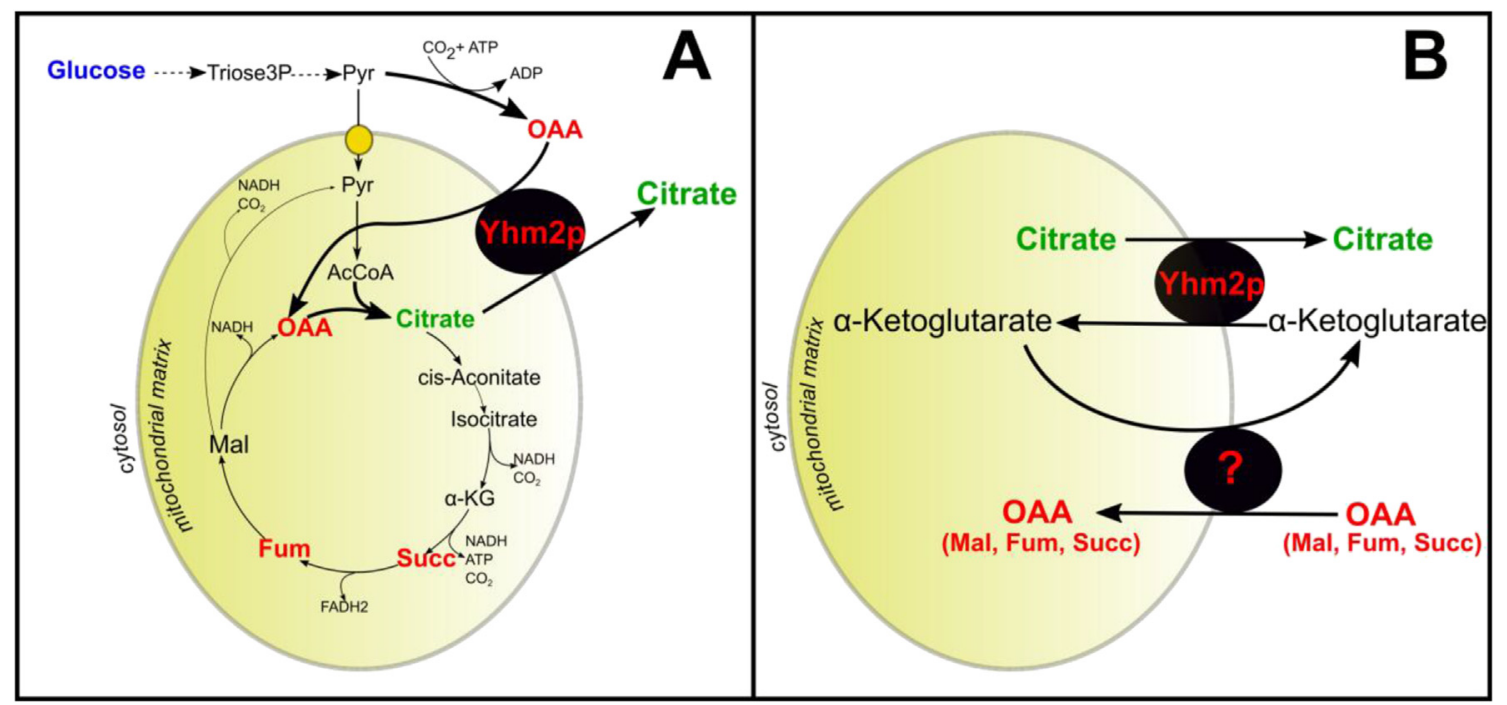

Fig. 6. Schematic illustration of the reactions catalyzed by YlYhm2p. The export of citrate in exchange for cytosolic oxaloacetate is shown in (A) and the citrate/ dicarboxylate shuttle in (B). The latter consists of a citrate ${ }_{\text {in }} / \alpha$-ketoglutarate ${ }_{\text {out }}$ exchange, mediated by YlYhm2p, and an $\alpha$-ketoglutarate in $_{\text {inalate }} /$ mut $_{\text {ouchange }}$ mediated by a not yet identified mitochondrial carrier in Y. lipolytica. (For interpretation of the references to colour in this figure legend, the reader is referred to the Web version of this article.) 


\section{Conflicts of interest}

The authors declare that they have no conflicts of interest with the contents of this article.

\section{Acknowledgements}

The work was carried out using the equipment of the Unique Scientific Facility of BRC VKPM with technical support of the Centre for Collective Use of NRC "Kurchatov Institute" - GosNIIgenetika and with financial support of Russian Federation (state task No. 595-00003-19 PR) from the one side, as well as using the equipment of the Department of Biosciences, Biotechnologies and Biopharmaceutics, University of Bari "Aldo Moro" and the CNR Institute IBIOM from the other side.

\section{Appendix A. Supplementary data}

Supplementary data to this article can be found online at https:// doi.org/10.1016/j.ymben.2019.05.002.

\section{References}

Agrimi, G., Russo, A., Pierri, C.L., Palmieri, F., 2012a. The peroxisomal NAD + carrier of Arabidopsis thaliana transports coenzyme A and its derivatives. J. Bioenerg. Biomembr. 44, 333-340.

Agrimi, G., Russo, A., Scarcia, P., Palmieri, F., 2012b. The human gene SLC25A17 encodes a peroxisomal transporter of coenzyme A, FAD and NAD +. Biochem. J. 443, 241-247.

Barth, G., Gaillardin, C., 1997. Physiology and genetics of the dimorphic fungus Yarrowia lipolytica. FEMS Microbiol. Rev. 19, 219-237.

Beopoulos, A., Nicaud, J.M., Gaillardin, C., 2011. An overview of lipid metabolism in yeasts and its impact on biotechnological processes. Appl. Microbiol. Biotechnol. 90, 1193-1206.

Bisaccia, F., De Palma, A., Dierks, T., Krämer, R., Palmieri, F., 1993. Reaction mechanism of the reconstituted tricarboxylate carrier from rat liver mitochondria. Biochim. Biophys. Acta Bioenerg. 1142, 139-145.

Bisaccia, F., De Palma, A., Palmieri, F., 1989. Identification and purification of the tricarboxylate carrier from rat liver mitochondria. Biochim. Biophys. Acta Bioenerg. 977, 171-176.

Bisaccia, F., De Palma, A., Prezioso, G., Palmieri, F., 1990. Kinetic characterization of the reconstituted tricarboxylate carrier from rat liver mitochondria. Biochim. Biophys. Acta Bioenerg. 1019, 250-256.

Bisaccia, F., Palmieri, F., 1984. Specific elution from hydroxylapatite of the mitochondrial phosphate carrier by cardiolipin. Biochim. Biophys. Acta Bioenerg. 766, 386-394.

Blazeck, J., Hill, A., Jamoussi, M., Pan, A., Miller, J., Alper, H.S., 2015. Metabolic engineering of Yarrowia lipolytica for itaconic acid production. Metab. Eng. 32, 66-73.

Boulton, C.A., Ratledge, C., 1981. Correlation of lipid accumulation in yeasts with possession of ATP: citrate lyase. Microbiology 127, 169-176.

Castegna, A., Scarcia, P., Agrimi, G., Palmieri, L., Rottensteiner, H., Spera, I., Germinario, L., Palmieri, F., 2010. Identification and functional characterization of a novel mitochondrial carrier for citrate and oxoglutarate in Saccharomyces cerevisiae. J. Biol. Chem. 285, 17359-17370.

Cavallo, E., Charreau, H., Cerrutti, P., Foresti, M.L., 2017. Yarrowia lipolytica: a model yeast for citric acid production. FEMS Yeast Res. 17 fox084.

Di Noia, M.A., Todisco, S., Cirigliano, A., Rinaldi, T., Agrimi, G., Iacobazzi, V., Palmieri, F., 2014. The human SLC25A33 and SLC25A36 genes of solute carrier family 25 encode two mitochondrial pyrimidine nucleotide transporters. J. Biol. Chem. 289, 33137-33148.

Dulermo, T., Lazar, Z., Dulermo, R., Rakicka, M., Haddouche, R., Nicaud, J.M., 2015. Analysis of ATP-citrate lyase and malic enzyme mutants of Yarrowia lipolytica points out the importance of mannitol metabolism in fatty acid synthesis. Biochim. Biophys. Acta 1851, 1107-1117.

Fiermonte, G., Walker, J.E., Palmieri, F., 1993. Abundant bacterial expression and reconstitution of an intrinsic membrane-transport protein from bovine mitochondria. Biochem. J. 294 (Pt 1), 293-299.

Folch, J., Lees, M., Sloane Stanley, G.H., 1957. A simple method for the isolation and purification of total lipides from animal tissues. J. Biol. Chem. 226, 497-509.

Gibson, D.G., Young, L., Chuang, R.Y., Venter, J.C., Hutchison 3rd, C.A., Smith, H.O., 2009. Enzymatic assembly of DNA molecules up to several hundred kilobases. Nat. Methods 6, 343-345.

Guo, H., Madzak, C., Du, G., Zhou, J., 2016. Mutagenesis of conserved active site residues of dihydrolipoamide succinyltransferase enhances the accumulation of $\alpha$-ketoglutarate in Yarrowia lipolytica. Appl. Microbiol. Biotechnol. 100, 649-659.

Hoyos, M.E., Palmieri, L., Wertin, T., Arrigoni, R., Polacco, J.C., Palmieri, F., 2003. Identification of a mitochondrial transporter for basic amino acids in Arabidopsis thaliana by functional reconstitution into liposomes and complementation in yeast. Plant J. 33, 1027-1035.

Indiveri, C., Iacobazzi, V., Giangregorio, N., Palmieri, F., 1998. Bacterial overexpression, purification, and reconstitution of the carnitine/acylcarnitine carrier from rat liver mitochondria. Biochem. Biophys. Res. Commun. 249, 589-594.

Kamzolova, S.V., Shamin, R.V., Stepanova, N.N., Morgunov, G.I., Lunina, J.N., Allayarov, R.K., Samoilenko, V.A., Morgunov, I.G., 2018. Fermentation conditions and media optimization for isocitric acid production from ethanol by Yarrowia lipolytica. BioMed Res. Int. 2018, 2543210.

Kaplan, R., Mayor, J., Johnston, N., Oliveira, D., 1990. Purification and characterization of the reconstitutively active tricarboxylate transporter from rat liver mitochondria. J. Biol. Chem. 265, 13379-13385.

Kaplan, R.S., Mayor, J.A., Gremse, D.A., Wood, D.O., 1995. High level expression and characterization of the mitochondrial citrate transport protein from the yeast Saccharomyces cerevisiae. J. Biol. Chem. 270, 4108-4114.

Kirimura, K., Kobayashi, K., Yoshioka, I., 2019. Decrease of citric acid produced by Aspergihllus niger through disruption of the gene encoding a putative mitochondrial citrate-oxoglutarate shuttle protein. Biosci. Biotechnol. Biochem. 1-9.

Klingenberg, M., 2008. The ADP and ATP transport in mitochondria and its carrier. Biochim. Biophys. Acta Biomembr. 1778, 1978-2021.

Krämer, R., Palmieri, F., 1992. Metabolite Carriers in Mitochondria. New Comprehensive Biochemistry, vol. 23. Elsevier, pp. 359-384.

Kretzschmar, A., Otto, C., Holz, M., Werner, S., Hubner, L., Barth, G., 2013. Increased homologous integration frequency in Yarrowia lipolytica strains defective in nonhomologous end-joining. Curr. Genet. 59, 63-72.

Ledesma-Amaro, R., Dulermo, R., Niehus, X., Nicaud, J.M., 2016. Combining metabolic engineering and process optimization to improve production and secretion of fatty acids. Metab. Eng. 38, 38-46.

Li, X., Wang, P., Ge, Y., Wang, W., Abbas, A., Zhu, G., 2013. NADP(+)-specific isocitrate dehydrogenase from oleaginous yeast Yarrowia lipolytica CLIB122: biochemical characterization and coenzyme sites evaluation. Appl. Biochem. Biotechnol. 171, 403-416.

Liu, H.H., Ji, X.J., Huang, H., 2015. Biotechnological applications of Yarrowia lipolytica: past, present and future. Biotechnol. Adv. 33, 1522-1546.

Luevano-Martinez, L.A., Moyano, E., de Lacoba, M.G., Rial, E., Uribe-Carvajal, S., 2010 Identification of the mitochondrial carrier that provides Yarrowia lipolytica with a fatty acid-induced and nucleotide-sensitive uncoupling protein-like activity. Biochim. Biophys. Acta 1797, 81-88.

Madzak, C., 2015. Yarrowia lipolytica: recent achievements in heterologous protein expression and pathway engineering. Appl. Microbiol. Biotechnol. 99, 4559-4577.

Madzak, C., Treton, B., Blanchin-Roland, S., 2000. Strong hybrid promoters and integrative expression/secretion vectors for quasi-constitutive expression of heterologous proteins in the yeast Yarrowia lipolytica. J. Mol. Microbiol. Biotechnol. 2, 207-216.

Marobbio, C., Agrimi, G., Lasorsa, F., Palmieri, F., 2003. Identification and functional reconstitution of yeast mitochondrial carrier for S-adenosylmethionine. EMBO J. 22, 5975-5982.

Marobbio, C.M., Giannuzzi, G., Paradies, E., Pierri, C.L., Palmieri, F., 2008, $\alpha-$ Isopropylmalate, a leucine biosynthesis intermediate in yeast, is transported by the mitochondrial oxalacetate carrier. J. Biol. Chem. 283, 28445-28453.

Marobbio, C.M.T., Di Noia, M.A., Palmieri, F., 2006. Identification of a mitochondrial transporter for pyrimidine nucleotides in Saccharomyces cerevisiae: bacterial expression, reconstitution and functional characterization. Biochem. J. 393, 441-446.

Mentel, M., Piskur, J., Neuveglise, C., Rycovska, A., Cellengova, G., Kolarov, J., 2005. Triplicate genes for mitochondrial ADP/ATP carriers in the aerobic yeast Yarrowia lipolytica are regulated differentially in the absence of oxygen. Mol. Genet. Genom. Mol. Gen. Genet. 273, 84-91.

Monné, M., Daddabbo, L., Gagneul, D., Obata, T., Hielscher, B., Palmieri, L., Miniero, D.V., Fernie, A.R., Weber, A.P., Palmieri, F., 2018. Uncoupling proteins 1 and 2 (UCP1 and UCP2) from Arabidopsis thaliana are mitochondrial transporters of aspartate, glutamate and dicarboxylates. J. Biol. Chem. 293, 4213-4227.

Monne, M., Miniero, D.V., Obata, T., Daddabbo, L., Palmieri, L., Vozza, A., Nicolardi, M.C., Fernie, A.R., Palmieri, F., 2015. Functional characterization and organ distribution of three mitochondrial ATP-Mg/Pi carriers in Arabidopsis thaliana. Biochim. Biophys. Acta Bioenerg. 1847, 1220-1230.

Morounov, I.G., Kamzolova, S.V., Lunina, J.N., 2013. The citric acid production from raw glycerol by Yarrowia lipolytica yeast and its regulation. Appl. Microbiol. Biotechnol. 97, 7387-7397.

Nicaud, J.M., 2012. Yarrowia lipolytica. Yeast 29, 409-418.

Palmieri, F., 1994. Mitochondrial carrier proteins. FEBS Lett. 346, 48-54.

Palmieri, F., 2004. The mitochondrial transporter family (SLC25): physiological and pathological implications. Pflügers Archiv 447, 689-709.

Palmieri, F., 2013. The mitochondrial transporter family SLC25: identification, properties and physiopathology. Mol. Aspect. Med. 34, 465-484.

Palmieri, F., 2014. Mitochondrial transporters of the SLC25 family and associated diseases: a review. J. Inherit. Metab. Dis. 37, 565-575.

Palmieri, F., Indiveri, C., Bisaccia, F., Iacobazzi, V., 1995. [25] Mitochondrial Metabolite Carrier Proteins: Purification, Reconstitution, and Transport Studies. Methods in Enzymology, vol. 260. Elsevier, pp. 349-369.

Palmieri, F., Monné, M., 2016. Discoveries, metabolic roles and diseases of mitochondrial carriers: a review. Biochim. Biophys. Acta Mol. Cell Res. 1863, 2362-2378.

Palmieri, F., Pierri, C.L., 2010. Mitochondrial metabolite transport. Essays Biochem. 47, $37-52$.

Palmieri, F., Prezioso, G., Quagliariello, E., Klingenberg, M., 1971. Kinetic study of the dicarboxylate carrier in rat liver mitochondria. Eur. J. Biochem. 22, 66-74.

Palmieri, F., Stipani, I., Quagliariello, E., Klingenberg, M., 1972. Kinetic study of the tricarboxylate carrier in rat liver mitochondria. FEBS J. 26, 587-594.

Palmieri, L., Agrimi, G., Runswick, M.J., Fearnley, I.M., Palmieri, F., Walker, J.E., 2001. Identification in Saccharomyces cerevisiae of two isoforms of a novel mitochondrial transporter for 2-oxoadipate and 2-oxoglutarate. J. Biol. Chem. 276, 1916-1922. 
Palmieri, L., Lasorsa, F.M., Iacobazzi, V., Runswick, M.J., Palmieri, F., Walker, J.E., 1999a. Identification of the mitochondrial carnitine carrier in Saccharomyces cerevisiae. FEBS Lett. 462, 472-476.

Palmieri, L., Vozza, A., Hönlinger, A., Dietmeier, K., Palmisano, A., Zara, V., Palmieri, F., 1999b. The mitochondrial dicarboxylate carrier is essential for the growth of Saccharomyces cerevisiae on ethanol or acetate as the sole carbon source. Mol. Microbiol. 31, 569-577.

Papagianni, M., 2007. Advances in citric acid fermentation by Aspergillus niger: biochemical aspects, membrane transport and modeling. Biotechnol. Adv. 25, 244-263.

Pebay-Peyroula, E., Dahout-Gonzalez, C., Kahn, R., Trézéguet, V., Lauquin, G.J.-M., Brandolin, G., 2003. Structure of mitochondrial ADP/ATP carrier in complex with carboxyatractyloside. Nature 426, 39.

Picault, N., Palmieri, L., Pisano, I., Hodges, M., Palmieri, F., 2002. Identification of novel transporter for dicarboxylates and tricarboxylates in plant mitochondria: bacterial expression, reconstitution, functional characterization and tissue distribution. J. Biol. Chem. 277, 24204-24211.

Porcelli, V., Fiermonte, G., Longo, A., Palmieri, F., 2014. The human gene SLC25A29, of solute carrier family 25 , encodes a mitochondrial transporter of basic amino acids. J. Biol. Chem. 289, 13374-13384.

Pronk, J.T., Wenzel, T.J., Luttik, M.A., Klaassen, C.C., Scheffers, W.A., Steensma, H.Y., van Dijken, J.P., 1994. Energetic aspects of glucose metabolism in a pyruvate-dehydrogenase-negative mutant of Saccharomyces cerevisiae. Microbiology 140 (Pt 3) 601-610.

Qiao, K., Wasylenko, T.M., Zhou, K., Xu, P., Stephanopoulos, G., 2017. Lipid production in Yarrowia lipolytica is maximized by engineering cytosolic redox metabolism. Nat Biotechnol. 35, 173.

Ruprecht, J.J., Hellawell, A.M., Harding, M., Crichton, P.G., McCoy, A.J., Kunji, E.R., 2014. Structures of yeast mitochondrial ADP/ATP carriers support a domain-based alternating-access transport mechanism. Proc. Natl. Acad. Sci. Unit. States Am. 111, E426-E434.

Sambrook, J., Maniatis, T., Fritsch, E., 1989. Molecular Cloning: a Laboratory Manual. Cold Spring Harbor Laboratory, Cold Spring Harbor, N. Y.

Scarcia, P., Palmieri, L., Agrimi, G., Palmieri, F., Rottensteiner, H., 2017. Three mitochondrial transporters of Saccharomyces cerevisiae are essential for ammonium fixation and lysine biosynthesis in synthetic minimal medium. Mol. Genet. Metabol. 122, 54-60.

Steiger, M.G., Rassinger, A., Mattanovich, D., Sauer, M., 2019. Engineering of the citrate exporter protein enables high citric acid production in Aspergillus niger. Metab. Eng. 52, 224-231.

Tai, M., Stephanopoulos, G., 2013. Engineering the Push and Pull of Lipid Biosynthesis in Oleaginous Yeast Yarrowia Lipolytica for Biofuel Production. Metab. Eng. 15, 1-9.

Verbeke, J., Beopoulos, A., Nicaud, J.M., 2013. Efficient homologous recombination with short length flanking fragments in Ku70 deficient Yarrowia lipolytica strains. Biotechnol. Lett. 35, 571-576.

Wasylenko, T.M., Ahn, W.S., Stephanopoulos, G., 2015. The oxidative pentose phosphate pathway is the primary source of NADPH for lipid overproduction from glucose in Yarrowia lipolytica. Metab. Eng. 30, 27-39.

Xu, P., Qiao, K., Ahn, W.S., Stephanopoulos, G., 2016. Engineering Yarrowia lipolytica as a platform for synthesis of drop-in transportation fuels and oleochemicals. Proc. Natl. Acad. Sci. U. S. A. 113, 10848-10853.

Yuzbashev, T.V., Bondarenko, P.Y., Sobolevskaya, T.I., Yuzbasheva, E.Y., Laptev, I.A., Kachala, V.V., Fedorov, A.S., Vybornaya, T.V., Larina, A.S., Sineoky, S.P., 2016. Metabolic evolution and 13C flux analysis of a succinate dehydrogenase deficient strain of Yarrowia lipolytica. Biotechnol. Bioeng. 113, 2425-2432.

Yuzbashev, T.V., Yuzbasheva, E.Y., Sobolevskaya, T.I., Laptev, I.A., Vybornaya, T.V., Larina, A.S., Matsui, K., Fukui, K., Sineoky, S.P., 2010. Production of succinic acid at low $\mathrm{pH}$ by a recombinant strain of the aerobic yeast Yarrowia lipolytica. Biotechnol. Bioeng. 107, 673-682.

Yuzbasheva, E.Y., Mostova, E.B., Andreeva, N.I., Yuzbashev, T.V., Laptev, I.A., Sobolevskaya, T.I., Sineoky, S.P., 2017. Co-expression of glucose-6-phosphate dehydrogenase and acyl-CoA binding protein enhances lipid accumulation in the yeast Yarrowia lipolytica. New Biotechnol. 39, 18-21.

Yuzbasheva, E.Y., Yuzbashev, T.V., Perkovskaya, N.I., Mostova, E.B., Vybornaya, T.V. Sukhozhenko, A.V., Toropygin, I.Y., Sineoky, S.P., 2015. Cell surface display of Yarrowia lipolytica lipase Lip2p using the cell wall protein YlPir1p, its characterization, and application as a whole-cell biocatalyst. Appl. Biochem. Biotechnol. 175, 3888-3900. 